*For correspondence:

\title{
Formation of the substantia nigra requires Reelin-mediated fast, laterally-directed migration of dopaminergic neurons
}

\section{Ankita Ravi Vaswani ${ }^{1}$, Beatrice Weykopf ${ }^{2}$, Cathleen Hagemann ${ }^{1}$, Hans-Ulrich Fried $^{3}$, Oliver Brüstle ${ }^{2}$, Sandra Blaess ${ }^{1}$}

$7 \quad{ }^{1}$ Neurodevelopmental Genetics, Institute of Reconstructive Neurobiology,

Sigmund-Freud-Str. 25, Bonn, 53127, Germany; ${ }^{2}$ Institute of Reconstructive Neurobiology,

Sigmund-Freud-Str. 25, Bonn, 53127, Germany; ${ }^{3}$ Light Microscope Facility, German

Center for Neurodegenerative Diseases (DZNE), Sigmund-Freud-Str. 27, Bonn, 53127, Germany

Abstract Midbrain dopaminergic (mDA) neurons migrate to form the laterally-located substantia nigra pars compacta (SN) and medially-located ventral tegmental area (VTA), but little is known about the underlying cellular and molecular processes. Reelin signaling regulates tangential migration of SN-mDA neurons, but whether Reelin acts directly on SN-mDA neurons and how it affects their cellular morphology and migratory behavior has not been explored. Here we visualize the dynamic cell morphologies of tangentially migrating SN-mDA neurons with 3D-time-lapse imaging and identify two distinct migration modes. Slow migration is the default mode in SN-mDA neurons, while fast, laterally-directed migration occurs infrequently and is strongly associated with bipolar cell morphology. By specifically inactivating Reelin signaling in mDA neurons we demonstrate its direct role in SN-mDA tangential migration. We show that Reelin signaling promotes laterally-biased movements in mDA neurons during their slow migration mode, stabilizes leading process morphology and increases the probability of fast, laterally-directed migration.

\section{Introduction}

Dopaminergic neurons in the ventral midbrain (mDA neurons) are the major source of dopamine in the mammalian brain. Dysfunction in the dopaminergic system is associated with schizophrenia, addiction and depression, and degeneration of mDA neurons in the substantia nigra pars compacta (SN) results in the motor symptoms of Parkinson's disease Grace and Bunney (1980), Volkow and Morales (2015), Przedborski (2017). mDA neurons originate in the floor plate of the ventral mesencephalon, from where they migrate to cluster into the laterally-positioned SN, the mediallylocated ventral tegmental area (VTA) and the posterior retrorubral field. SN-mDA neurons project predominantly to the dorsal striatum and modulate voluntary movement Weisenhorn et al. (2016), while VTA-mDA neurons project to various forebrain targets, including the prefrontal cortex, nucleus accumbens and basolateral amygdala, and are important for the regulation of cognitive function and reward behavior Morales and Margolis (2017). How this anatomy is set up during development remains unclear.

mDA neurons differentiation starts at embryonic day (E) 10.5 in the mouse, when the first mDA 
neurons that express tyrosine hydroxylase $(\mathrm{TH})$, the rate limiting enzyme in dopamine synthesis and a marker for differentiated mDA neurons, leave the ventricular zone of the ventral midbrain. Differentiated mDA neurons undergo a maturation process as they migrate to reach their final positions Blaess and Ang (2015). We have previously shown that both SN- and VTA-mDA neurons undergo radial migration into the mantle layer of the developing ventral midbrain where they remain intermingled until E13.5. Between E13.5 and E15.5, mDA neurons destined for the SN migrate tangentially to more lateral positions, resulting in the segregation of mDA neurons into the laterally-located SN and the medially-situated VTA Bodea et al. (2014). This particular migration pattern suggests that SN-mDA neurons have the specific molecular machinery to respond to cues in their environment that direct their lateral migration. As exemplified by migration studies in cortical brain areas, a comprehensive characterization of migratory modes and accompanying changes in cell morphology is indispensable for unraveling the molecular mechanisms by which cell-type specific migratory behavior is regulated Kriegstein and Noctor (2004). So far, a detailed understanding of mDA neuronal migratory behavior has remained elusive due to challenges in visualizing migrating mDA neurons in sufficient detail.

At the molecular level, Reelin, an extracellular matrix molecule and known regulator of neuronal migration in various brain areas, is essential for the correct lateral localization of SN-mDA neurons. Reelin binds to its receptors APOER2 and VLDLR, and induces the phosphorylation of the intracellular transducer DAB1 Hiesberger et al. (1999), Trommsdorff et al. (1999). Phosphorylated DAB1 then mediates Reelin signaling by regulating cell adhesive properties or cytoskeletal stability Chai et al. (2016), Franco et al. (2011), Howell et al. (1997). In mice homozygous for null alleles of Reelin (reeler) or Dab1 (scrambler or Dab1null), in Vldlr/Apoer2 double knockout mice, or in organotypic slices in which Reelin signaling is blocked, SN-mDA neurons do not reach their final positions in the ventrolateral midbrain and accumulate instead in the area of the lateral VTA Bodea et al. (2014), Vaswani and Blaess (2016) Kang et al. (2010); Nishikawa et al. (2003); Sharaf et al. (2013) . Whether Reelin affects tangential (lateral) mDA neuronal migration directly, or whether the failure of $\mathrm{SN}-\mathrm{mDA}$ neurons to reach their final position in Reelin pathway mutants is due to alterations in glia fibers or neighboring neuronal populations has not been explored. Moreover, it is not understood how the loss of Reelin signaling alters dynamic migration processes of mDA neurons and which of the multiple signaling events downstream of Reelin plays a role in mDA neuronal migration.

Here, we dissect the complex dynamic morphological changes that underlie the tangential migration of SN-mDA neurons using 2-photon excitation time-lapse imaging and a semi-automated data analysis pipeline. We find that mDA neurons migrate in two modes: infrequent laterallydirected fast migration and frequent slow movement. We demonstrate that migrating mDA neurons undergo dynamic changes in cell morphology and show that fast, directed migratory spurts are strongly associated with bipolar morphology. Combining conditional gene inactivation, genetic fate mapping and time-lapse imaging, we demonstrate that Reelin affects mDA neuronal migration in a direct manner and promotes fast, laterally-directed migration of mDA neurons and stabilizes their leading process morphology.

\section{Results}

\section{Reelin signaling acts directly on tangentially migrating mDA neurons}

As a first step to understand the regulation of MDA tangential migration by Reelin, we investigated whether Reelin signaling is directly required by mDA neurons for their correct lateral localization. We conditionally inactivated Dab1 in differentiated mDA neurons using a Cre-line in which Cre is knocked into the endogenous dopamine transporter (Dat) locus (genotype: Dat ${ }^{\mathrm{Cre} /+}$, Dab $1^{\mathrm{del} / \text { flox }}$; referred to as Dab1 CKO) Ekstrand et al. (2007), Franco et al. (2011) (Figure 1A). To determine the onset of Cre-mediated recombination in the $D A T^{\mathrm{Cre} /+}$ mouse line, we crossed $D A T^{\mathrm{Cre} /+}$ mice with an enhanced yellow fluorescent protein (YFP)-expressing reporter mouse line (Rosa26 $6^{\text {lox-stop-lox-EYFP }}$ ) Srinivas et al. (2001). We observed widespread YFP-expression in $\mathrm{TH}$-positive $\left(\mathrm{TH}^{+}\right)$cells in the lateral mDA neuron domain starting at E13.5 (Figure 1- Figure supplement 1). Immunostaining for DAB1 
A

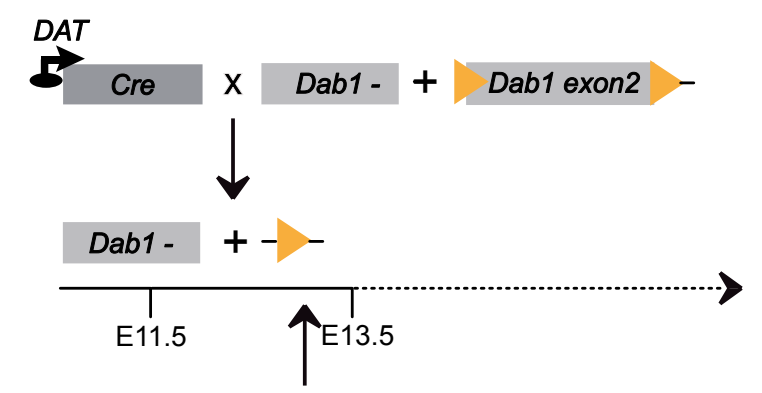

Conditional inactivation of Dab1 in mDA neurons after E13.5
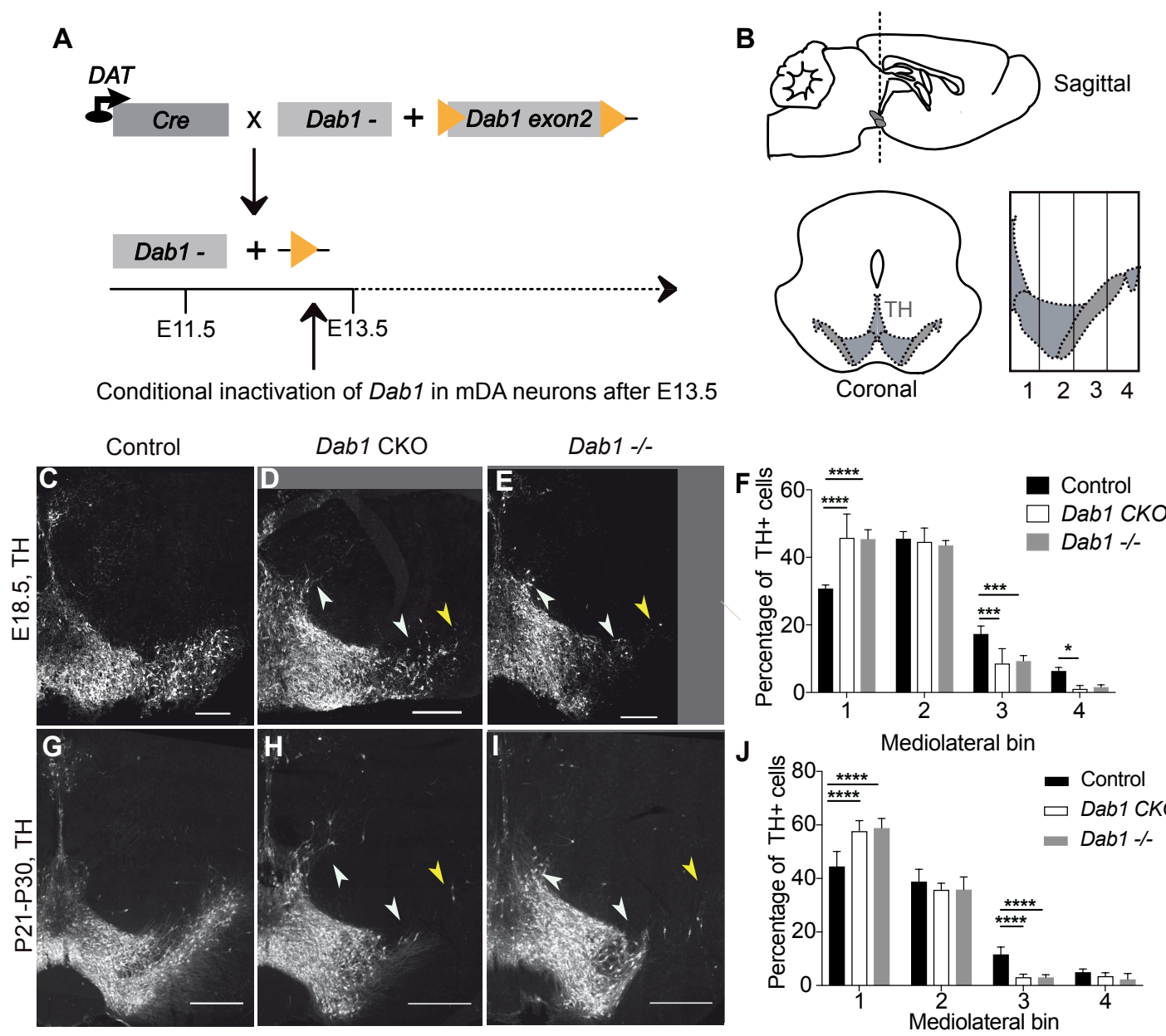

Figure 1. Direct role of Reelin signaling in tangential migration of mDA neurons. (A): Schematic showing Cre-mediated inactivation of Dab1 in mDA neurons. (B) Schematic representing the anteroposterior level of coronal sections used for the analysis, and the mediolateral grid used to quantify distribution of $\mathrm{TH}^{+}\left(\mathrm{Tyr}_{\mathrm{r}} \mathrm{sine}\right.$ Hydroxylase) neurons. (C-I) Immunostaining for TH and quantification of cell distribution for control, Dab1 CKO, and Dab1-/-midbrain regions at E18.5 (C-F) and at P21-P30 (G-J). White arrowheads indicate differences in the mediolateral distribution of $\mathrm{TH}^{+}$cells. Yellow arrowheads point to cells in the substantia nigra pars lateralis used as a landmark for the most lateral position in the mediolateral grids. (F,J) Quantification of mediolateral distribution of $\mathrm{TH}^{+}$cells for control, Dab1 CKO and Dab1-/-brains at E18.5 (F, n=4 for each genotype) and at P21-P30 (J, n=6 for each genotype). Data are represented as mean + s.e.m. **** indicates significant difference $p<0.0001$ as assessed by two-way ANOVA with Tukey's multiple comparison correction. Scale bars: (A-C) 100 $\mu \mathrm{m},(\mathrm{E}-\mathrm{G}) 200 \mu \mathrm{m}$. Figure 1- Figure supplement 1. Dat ${ }^{\text {Cre }}$ mediated recombination pattern.

Figure 1- Figure supplement 2. Specific loss of DAB1 protein in mDA neurons of Dab1 CKO brains.

Figure 1- Figure supplement 3. Mediolateral distribution of mDA neurons at E15.5 in absence of Reelin signaling.

at E15.5 showed that DAB1 was no longer expressed in the lateral $\mathrm{TH}^{+}$domain in Dab1 CKO mice, while it was still present in a non-dopaminergic area lateral to the SN (Figure 1- Figure supplement 2). Since the inactivation of Dab1 in Dab1 CKO mice occurs after radial migration of SN-mDA neurons is essentially completed Bodea et al. (2014), any defects observed in SN formation in this mouse model can be attributed to a misregulation of mDA tangential migration. Thus, the Dab1 CKO model allows us to specifically dissect out the direct role of Reelin signaling in the tangential migration of mDA neurons.

SN-mDA neurons fail to migrate to their correct lateral position in reeler, Dab1 null or Apoer2/VIdIr double knock-out mutants Bodea et al. (2014), Howell et al. (1997), Nishikawa et al. (2003). To examine whether this phenotype is recapitulated in Dab1 CKO mice, we compared the mediolateral 
distribution of $\mathrm{TH}^{+} \mathrm{mDA}$ neurons in coronal midbrain sections of control, Dab1 CKO and Dab1-/(genotype: Dab1 ${ }^{\mathrm{del} / \mathrm{del}}$ ) mice at postnatal day (P)21-P30 and embryonic time points (E15.5 and E18.5)(Figure 1; Figure 1- Figure supplement 3). We focused our analysis on intermediate rostrocaudal sections of the $\mathrm{TH}^{+} \mathrm{mDA}$ domain where the most severe defects in mediolateral distribution of mDA neurons are observed in reeler and Dab1-/- mice Bodea et al. (2014) (Figure 1B). In both the Dab1 CKO and Dab1-/- mice, mDA neurons failed to reach lateral positions in the SN and settled in more medial locations (Figure 1C-j; (Figure 1- Figure supplement 3). In addition, a few mDA neurons were aberrantly located dorsal to the VTA (Figure 1C-E, G-I). As the shift in the mediolateral

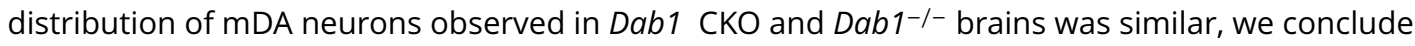
that Reelin acts directly on SN-mDA neurons to regulate their lateral migration.

We then asked whether such a direct function of Reelin is consistent with the localization of Reelin protein. During the time window of SN-mDA tangential migration (before E15.5), Reelin mRNA is expressed in the red nucleus, which is located dorsomedial to SN-mDA neurons. Whether Reelin protein is localized close to migrating SN-mDA neurons during this period has not been investigated Bodea et al. (2014), Nishikawa et al. (2003), Sharaf et al. (2015). Immunostaining for Reelin at E13.5 and E14.5 confirmed strong expression of the protein in the region of the red nucleus (Figure 2B,C,E,F). At E13.5 and E14.5, Reelin protein, but not Reelin mRNA, was also observed ventral and lateral to the red nucleus, including the area where the most lateral MDA neurons are localized at these stages (Figure 2A-G). Thus, the localization of Reelin protein at E13.5-E14.5 is consistent with a direct role of Reelin signaling in SN-mDA neuronal migration.

TH
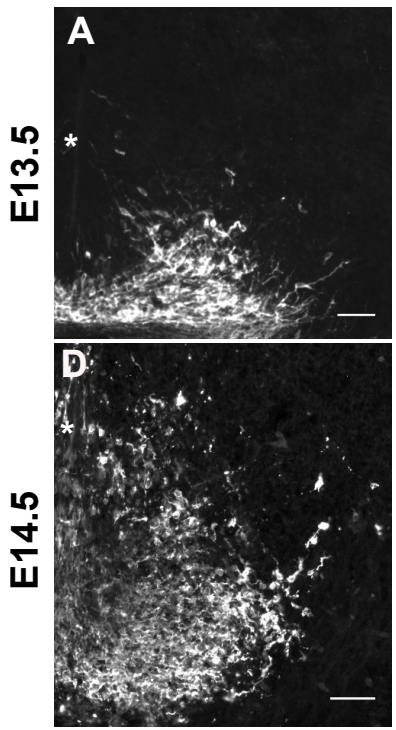

Reelin
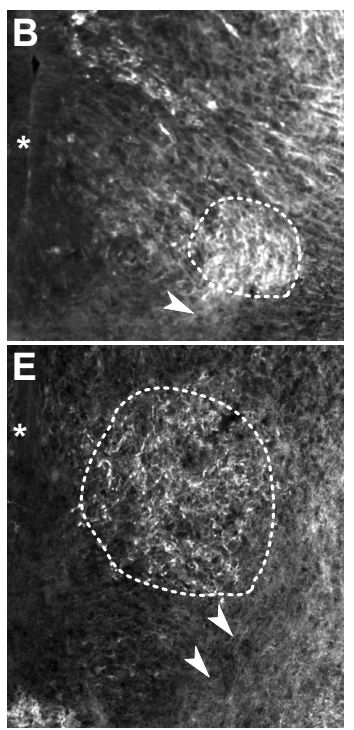

TH/Reelin
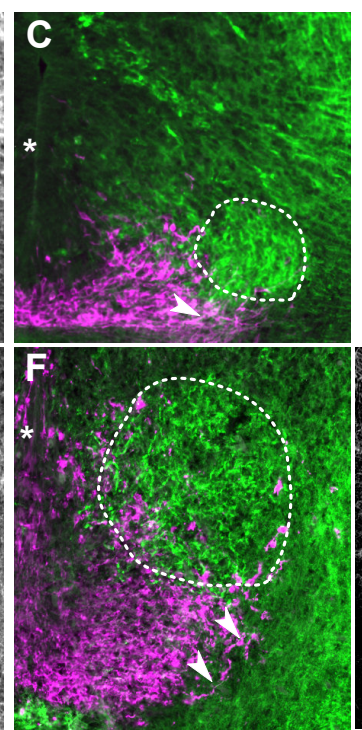

\section{Reelin mRNA}

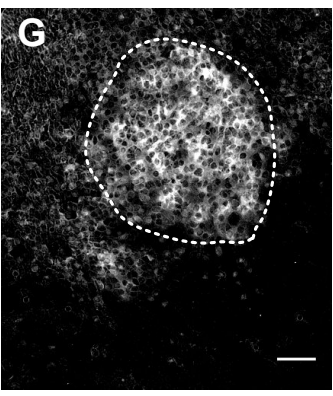

Figure 2. Reelin protein in the ventral midbrain at E13.5 and 14.5. (A-C) Double immunolabeling for TH and Reelin shows Reelin protein in the region of the red nucleus (RN, white dashed outline) and in the lateral $\mathrm{TH}^{+}$mDA domain (white arrowhead) at E13.5. (D-G) Double immunolabeling for TH and Reelin (D-F) and RNA in situ hybridization for Reelin mRNA (G) at E14.5. Reelin mRNA and Reelin protein are strongly expressed in the RN. Reelin protein is also localized ventral and lateral to the RN, overlapping with the lateral mDA domain (white arrowheads). Note that the colors in G were inverted. Asterisks indicate ventral midline. Scale bar: $50 \mu \mathrm{m}$.

\section{Reelin signaling contributes to the segregation of SN- and VTA-mDA neurons into sepa-}

\section{rate clusters}

Given that SN-mDA neurons fail to form the lateral SN in the absence of Reelin signaling, we asked whether Reelin signaling is important for the segregation of SN- and VTA-mDA neurons into separate clusters. We have previously shown that mDA neurons positive for the potassium channel GIRK2 (G-protein-regulated inward-rectifier potassium channel 2; expressed in mDA neurons in the SN and lateral VTA) are shifted medially in Dab1/- mice, while mDA neurons positive for 
Calbindin (expressed in VTA-mDA neurons and in a dorsal subset of SN-mDA neurons) are correctly localized Bodea et al. (2014), Björklund and Dunnett (2007) . Comparison of the mediolateral position of $\mathrm{TH}^{+}$, Calbindin ${ }^{+}$and $\mathrm{TH}^{+}, \mathrm{GIRK}^{+}$cells in control and Dab1 CKO brains at P30 showed that there was no significant difference in the distribution of $\mathrm{TH}^{+}, \mathrm{Calbindin}{ }^{+} \mathrm{mDA}$ neurons between Dab1 CKO mice and controls (data not shown). In contrast, the $\mathrm{TH}^{+}, \mathrm{GIRK}^{+} \mathrm{mDA}$ subpopulation showed a significant shift to a more medial position in the Dab1 CKO mice (Figure 3A-C). These results further confirmed that the Dab1 CKO phenotype recapitulates the phenotype observed in Dab1-1- mice.

To investigate the distribution of medially shifted SN-mDA neurons within the VTA we analyzed the expression of the transcription factor SOX6 (sex determining region Y-box6), and the Lim domain protein LMO3 (LIM domain only protein 3) as markers for SN-mDA neurons and the expression of the transcription factor OTX2 (Orthodenticle homeobox 2) in VTA-mDA neurons Salvio et al. (2010), Panman et al. (2014), Poulin et al. (2014), Bifsha et al. (2017), Manno et al. (2016). In E18.5 control brains, $\mathrm{TH}^{+}, \mathrm{OTX} 2^{+}$cells and $\mathrm{TH}^{+}$, SOX6 ${ }^{+}$cells were clearly separated at the boundary between SN and lateral VTA (Figure 3D,F). In Dab1 CKO mice, $\mathrm{TH}^{+}$, SOX6 ${ }^{+}$and $\mathrm{TH}^{+}, \mathrm{Lmo3}^{+} \mathrm{mDA}$ neurons were more medially located than in controls and were partially intermingled with $\mathrm{TH}^{+}$, OTX2 ${ }^{+}$mDA neurons (Figure 3D-I). Hence, the inactivation of Reelin signaling in mDA neurons results in an ectopic medial location of SN-mDA neurons and a partial mixing of the two populations at what would constitute the SN-lateral VTA border in control brains.

Time-lapse imaging of tangentially migrating mDA neurons reveals diverse migratory behaviors across a population of neurons, and in individual neurons across time

Having established the direct requirement of Reelin signaling in the tangential migration of SN-mDA neurons, we visualized their migration in the presence and absence of Reelin, thereby dissecting out the precise migratory behaviors regulated by Reelin signaling. To monitor mDA migration during development, sparse labeling of SN-mDA neurons is necessary to enable tracking and morphology analysis of their migration. We used an established genetic inducible fate mapping system to mosaically label SN-mDA progenitors and their descendants Blaess et al. (2011), Bodea et al. (2014) (Figure 4A). With this system, SN-mDA neurons are preferentially labeled and more than two-thirds of YFP-labeled neurons are $\mathrm{TH}^{+}$in the imaged regions at $\mathrm{E} 13.5$, and almost $90 \%$ are $\mathrm{TH}^{+}$at E14.5 Bodea et al. (2014). Henceforth, we refer to these YFP-labeled neurons as SN-mDA neurons.

Ex vivo horizontal organotypic slice cultures of the ventral brain from E13.5 embryos with mosaically labelled SN-mDA neurons were prepared for time-lapse imaging Bodea and Blaess (2012); Bodea et al. (2014) (Figure 4B). 2-photon excitation time-lapse microscopy allows 3D visualization of dynamic changes in cell morphologies of migrating SN-mDA neurons. As the migratory modes and associated changes in morphology of tangentially-migrating mDA neurons are unknown, we first defined migratory behavior in SN-mDA neurons using a number of parameters in slices of control mice and subsequently compared them with those of SN-mDA neurons in Dab ${ }^{1-/-}$ slices.

To characterize the whole range of migratory behaviors within the time window of imaging, we acquired 3D volume images of slices every 10 minutes, and tracked soma positions of a large number of neurons (806 neurons from 3 control slices, 844 neurons from 3 Dab1-/- slices). We then calculated speed and trajectory for each neuron's soma, at every time-point of imaging, based on location differences in consecutive volume images (Figure 4C-F, Movie 1). Plotting average speed distributions of cells from each slice, showed that the behavior of cells in different control slices and in different Dab1-/- slices was comparable (Figure 4- figure supplement 1A,B). However, individual cells' soma speeds varied considerably over time, and the maximum observed soma speed (henceforth max-speed) of a cell could be several times higher than its average speed (Figure 4F). Furthermore, ranking all control and all Dab1-/- cells by their max-speeds revealed great diversity as the max-speeds varied across cells in a smooth distribution from $183 \mu \mathrm{m} / \mathrm{hr}$ to $0 \mu \mathrm{m} / \mathrm{hr}$ for controls and from $134 \mu \mathrm{m} / \mathrm{hr}$ to $0 \mu \mathrm{m} / \mathrm{hr}$ for Dab1-/- cells (Figure 4- figure supplement 1C,D).

Two modes of tangential migration in SN-mDA neurons: frequent, slow movements and 

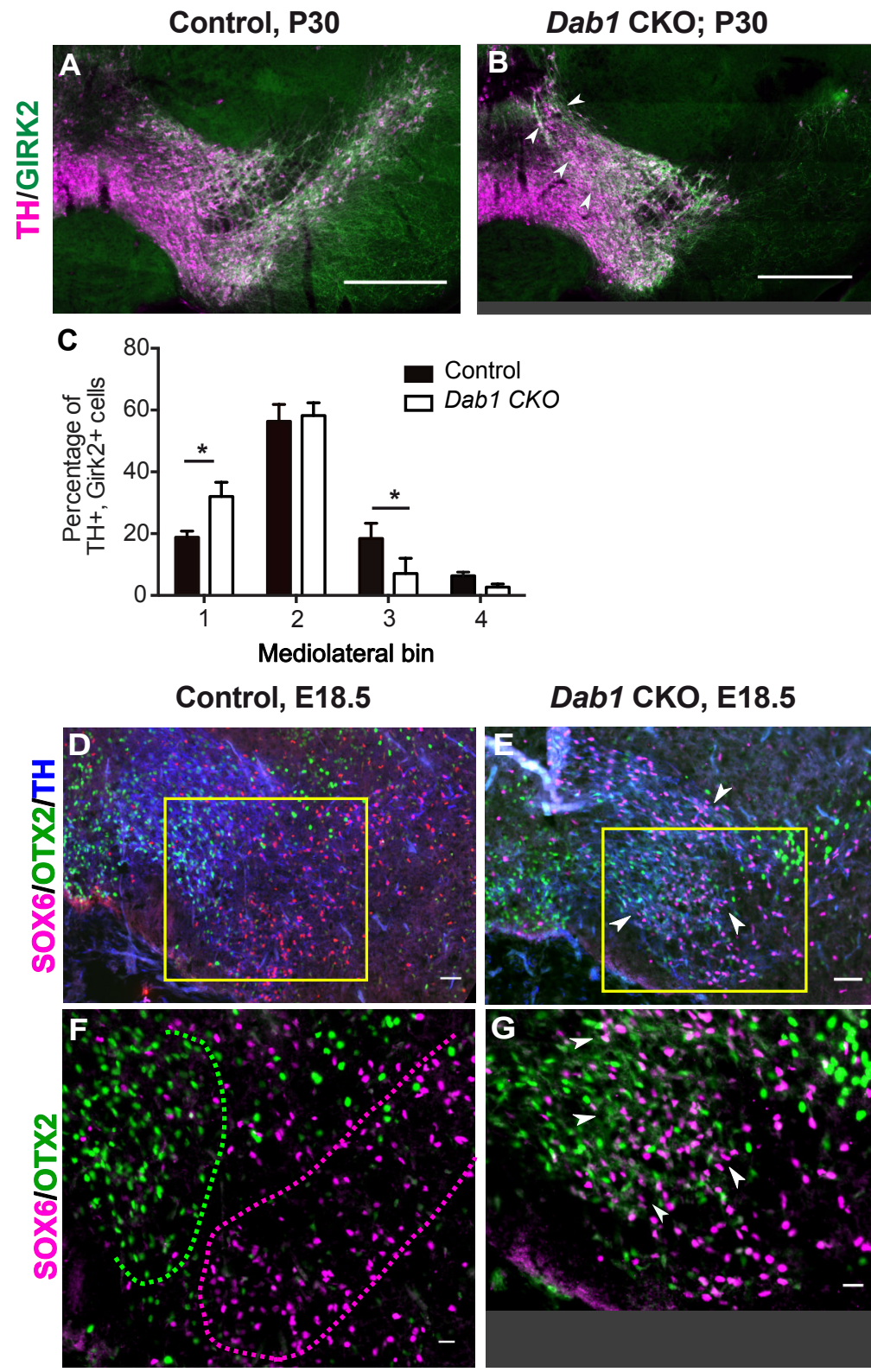

\section{Dab1 CKO, E18.5}
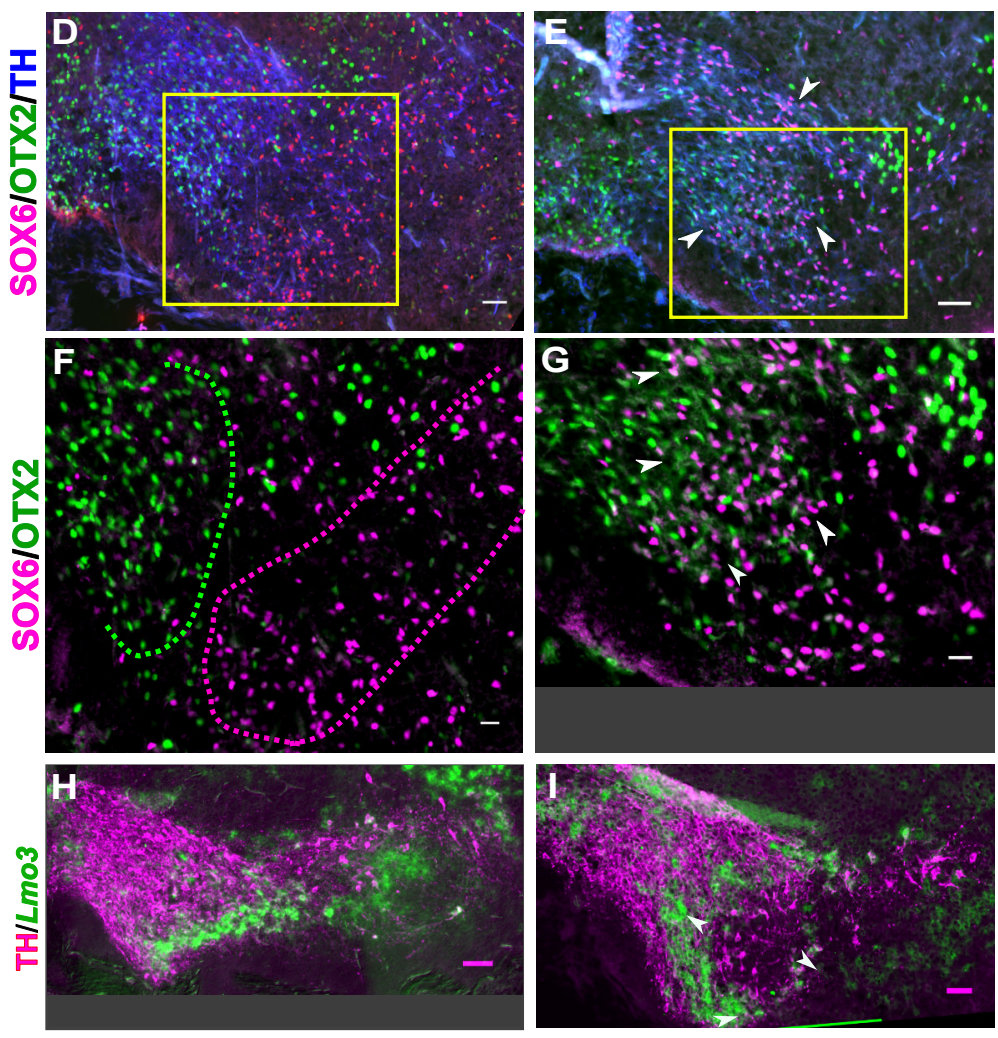

Figure 3. SN-mDA neurons do not completely segregate from VTA-mDA neurons in the absence of Reelin signaling. (A-C) Immunostaining for TH and GIRK2 in P30 Dab1 CKO mice. White arrowheads: medial accumulation of $\mathrm{TH}^{+}$, Girk2 ${ }^{+}$cells in Dab1 CKO (B) compared to control littermates (A). $\mathrm{TH}^{+}$, Girk2 ${ }^{+}$cells are shifted medially in Dab1 CKO brains compared to controls (C). * $p<0.05$; Student's t-test corrected for multiple comparisons (Holm-Sidak method) for $n=3$ brains/genotype. (D,E) Immunostaining for SOX6 (magenta), OTX2 (green) and TH (blue) on E18.5 control and Dab1 CKO brains. White arrowheads indicate altered distribution of $\mathrm{TH}^{+}$, SOX6 ${ }^{+}$cells in Dab1 CKO mice. Yellow boxes indicate regions presented in $\mathrm{F}$ and $\mathrm{G}$. $(\mathrm{F}, \mathrm{G})$ Higher zoom of $\mathrm{TH}^{+}$lateral VTA region in (D,E). In controls, $\mathrm{SOX6}^{+}$cells (dashed magenta line) and OTX2+ cells (dashed green line) are localized to separate regions (F). In Dab1 CKO, SOX6+ cells accumulate medially and are partially intermingled with OTX2 ${ }^{+}$cells (white arrowheads) $(\mathrm{G})$. (H,I) Immunostaining for TH and RNA in situ hybridization for $\mathrm{Lmo} 3$ shows ectopic medial localization of $\mathrm{TH}^{+}, \mathrm{Lmo}^{+}$cells in Dab1 CKO (white arrowheads in I). $\mathrm{Lmo} 3$ signal was inverted and false-colored in green. Cells in which Lmo3 was detected, show weak TH immunostaining, as the strong in situ hybridization signal interferes with antibody binding. Scale bars: $(A, B) 200$ $\mu \mathrm{m},(\mathrm{D}, \mathrm{E}, \mathrm{H}, \mathrm{I}) 50 \mu \mathrm{m},(\mathrm{F}, \mathrm{G}) 25 \mu \mathrm{m}$. 
A

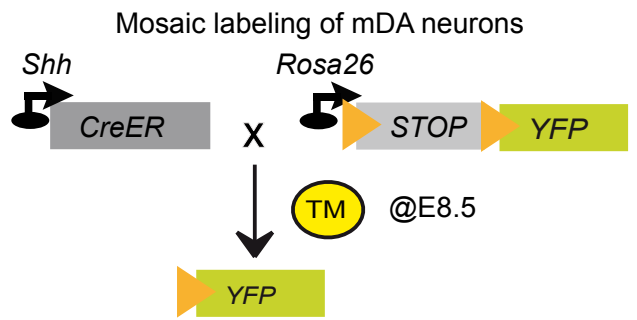

C

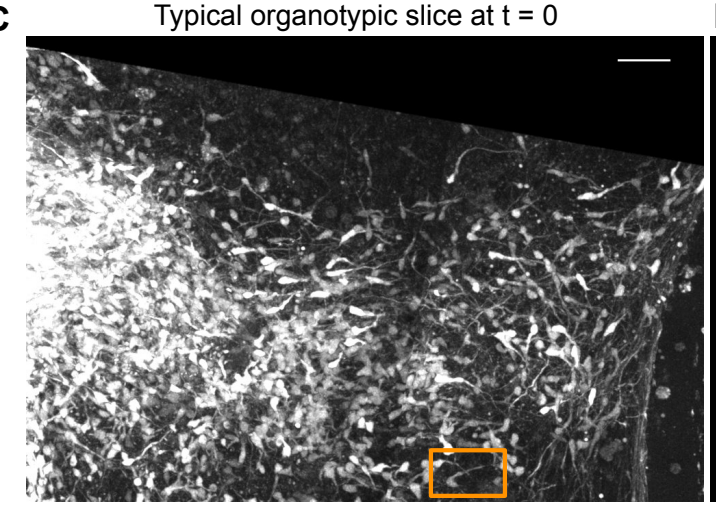

B

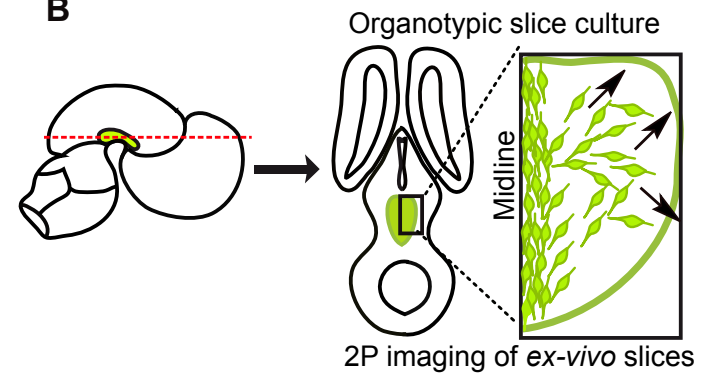

D

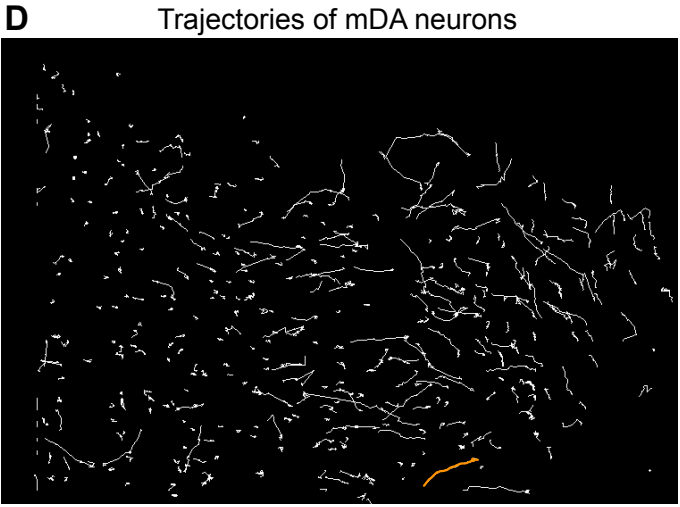

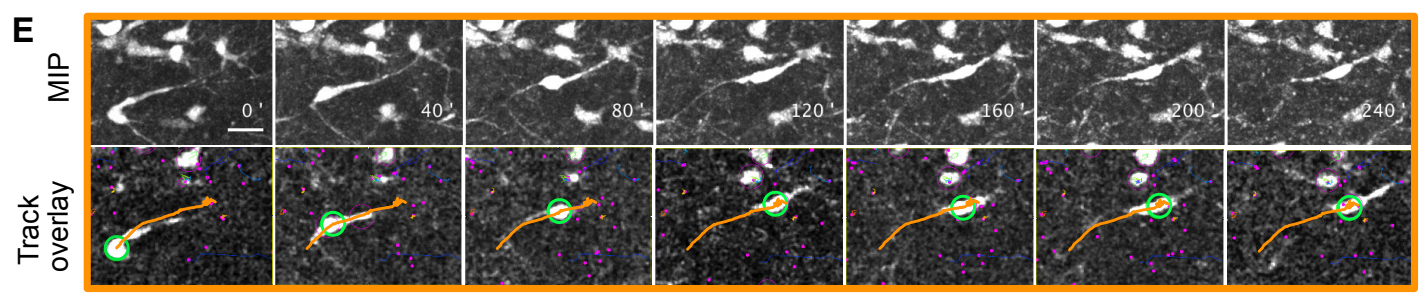

$\mathbf{F}$

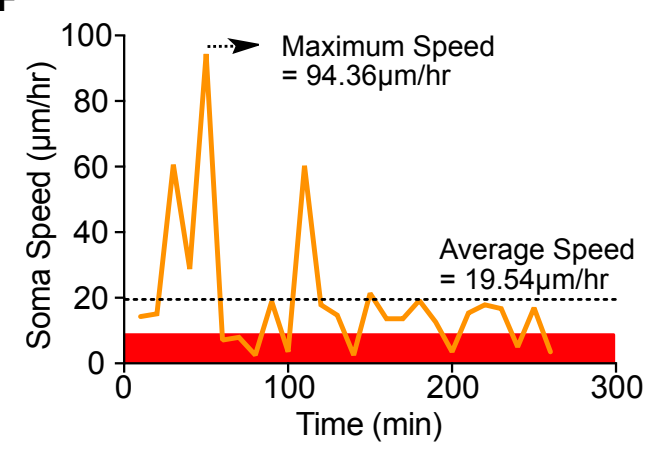

Figure 4. Visualizing mDA tangential migration with 2-photon excitation microscopy. (A) Schematic of the inducible genetic fate mapping system used to mosaically label mDA neurons by administering tamoxifen (TM) at E8.5. Shh: Sonic Hedgehog; YFP: yellow fluorescent protein. CreER: gene encoding a CRE-Estrogen Receptor fusion protein. (B) Schematic of horizontal organotypic slice culture preparations. Green regions represent location of mDA neurons in the embryonic brains (left) and horizontal slices (right). Red dashed line indicates dorsoventral level of slices. Black arrows indicate direction of tangential mDA migration. (C) Maximum intensity projection (MIP) of a control slice at $t=0$. Orange rectangle indicates location of cell shown in (E). (D) Trajectories of tracked neurons in slice shown in (C) after imaging for $270 \mathrm{~min}$. Trajectory in orange represents trajectory of neuron in (E). (E) MIP of time-lapse images show soma and processes of a tangentially migrating cell. Track overlays show the position of the soma (green circle) and trajectory of the cell (orange line) analyzed with the semi-automated tracking plugin TrackMate in Fiji. Magenta dots and circles represent tracked soma of close-by cells at different z-levels. (F) Speed profile of cell in (E) shows large variations in speed over time, with a maximum speed (dashed arrow) that is much higher than the average speed (dashed horizontal line). Rest phase (soma speed less than $10 \mu \mathrm{m} / \mathrm{hr}$ ) is indicated in red. Scale bars: (C,D) $50 \mu \mathrm{m}$, (E) $20 \mu \mathrm{m}$.

Figure 4 - Figure supplement 1. Average speed distributions and speed profiles of SN-mDA neurons. 


\section{infrequent, fast movements that are promoted by Reelin signaling}

The role of Reelin signaling has been studied extensively in the cortex and hippocampus. However, only few studies have examined Reelin function in regulating the speed of migrating neurons. These studies have shown that the effect of Reelin varies depending on the brain region and type of neuron analyzed Simó et al. (2010); Britto et al. (2013), Britto et al. (2011), Wang et al. (2018). We have previously demonstrated that inhibiting Reelin in ex vivo slices results in a decrease in average speed of SN-mDA neurons over long periods of imaging Bodea et al. (2014). In our current analysis we found no significant difference in the distribution of average speeds of the

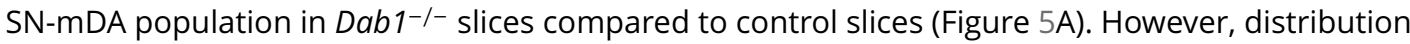
of max-speeds was significantly shifted towards lower speeds in the absence of Reelin signaling (control: $25^{\text {th }}$ percentile $=12.4 \mu \mathrm{m} / \mathrm{hr}$, median $=23.6 \mu \mathrm{m} / \mathrm{hr}, 75^{\text {th }}$ percentile $=48.1 \mu \mathrm{m} / \mathrm{hr}$, maximum $=183 \mu \mathrm{m} / \mathrm{hr} ;$ Dab1-/-: $25^{\text {th }}$ percentile $=10.1 \mu \mathrm{m} / \mathrm{hr}$, median $=15 \mu \mathrm{m} / \mathrm{hr}, 75^{\text {th }}$ percentile $=29.8$ $\mu \mathrm{m} / \mathrm{hr}$, maximum $=133.7 \mu \mathrm{m} / \mathrm{hr}$ ) (Figure $5 \mathrm{~B}$ ).

We then asked whether this shift towards lower max-speeds in Dab1-/- SN-mDA neurons was accompanied by other changes in migratory behavior, or whether the neurons simply displayed lower max-speeds while maintaining the same migratory, directional and morphological characteristics as control SN-mDA neurons. To answer this question, we compared variation in soma speed over time, migratory direction and cell morphology of control and Dab1-/- mDA neurons with similar max-speeds. For this analysis, we divided control and Dab1/- neurons into four groups based on the lower and upper quartiles of the Dab1//- max-speed distribution. We defined these groups in the following manner: non-migratory cells with max-speeds of less than $10 \mu \mathrm{m} / \mathrm{hr}$ (control $=126 / 806$, Dab1-/- $=205 / 844$ ), 'slow' cells with max-speeds from 10-30 $\mu \mathrm{m} / \mathrm{hr}$ (control = 355/806, Dab1-/- = 430/844), 'moderate' cells with max-speeds from 30-60 $\mu \mathrm{m} / \mathrm{hr}$ (control = 186/806, Dab1 $1^{-/-}=139 / 844$ ) and 'fast' cells with max-speeds $>60 \mu \mathrm{m} / \mathrm{hr}$, control $=139 / 806$, Dab1 $^{-/-}$ $=70 / 844$ ) (Figure 5B). Non-migratory cells failed to move more than $1.7 \mu \mathrm{m}$ in any two consecutive frames of analysis and were not included into the further analysis. Thus, a lower percentage of $\mathrm{SN}-\mathrm{mDA}$ neurons reached moderate or fast migration speeds in Dab1-/- slices compared to controls, increasing the proportion of both non-migratory and 'slow' cells.

Next, we asked how frequently migrating SN-mDA neurons moved with soma speeds comparable to their max-speeds and whether the fraction of total time-points spent in high migratory speeds was different in control and Dab1-/- populations. To evaluate this, we used the criteria previously defined for max-speeds, but applied them to individual soma speeds for each cell at each time point. For example, we analyzed the fraction of time (percentage of total time-points) spent by each 'fast' cell with a soma speed of more than $60 \mu \mathrm{m} / \mathrm{hr}$ (fast migratory phase), 30-60 $\mu \mathrm{m} / \mathrm{hr}$ (moderate migratory phase), 10-30 $\mu \mathrm{m} / \mathrm{hr}$ (slow migratory phase) and less than $10 \mu \mathrm{m} / \mathrm{hr}$ (resting phase). In control slices, 'fast', 'moderate' and 'slow' cells spent a predominant fraction of time at rest (62.6 $+/-20 \% ; 68.5+/-18.2 \%, 85.7+/-11.1 \%$, respectively) and were frequently in a slow migratory phase $(26.8+/-17.4 \%, 25.1+/-16.3 \%, 14.2+/-11.1 \%$, respectively). 'Fast' and 'moderate' cells achieved the moderate migratory phase in only a few frames $(5.5+/-5.5 \%$ and $6.3+/-3.9$, respectively), and the fast migratory phase (only in 'fast' cells) was equally infrequent ( $5.5+/-2.2 \%$ ) (Figure 5 B, Figure 5 Figure supplement 1). The amount of time SN-mDA neurons of the same max-speed group spent in the resting phase or in the respective migratory phases was comparable between individual cells in control and Dab1-/- slices (Figure 5 - Figure supplement 1D-F).

In summary, these results demonstrate that SN-mDA migration has two distinct modes: a frequent slow migration phase seen in all migrating SN-mDA neurons and an infrequent moderateto-fast phase occurring in a subset of SN-mDA neurons. These phases are superimposed over frequent periods of rest. Reelin signaling increases the proportion of migratory mDA neurons and the likelihood of moderate-to-fast movements in migrating mDA neurons. As moderate-to-fast migratory phases are only attained in very few frames in our slices, the average speed distribution of SN-mDA neurons are however not changed in Dab1-/- compared to control slices.

The Reelin-promoted infrequent fast movements of mDA neurons contribute to large 

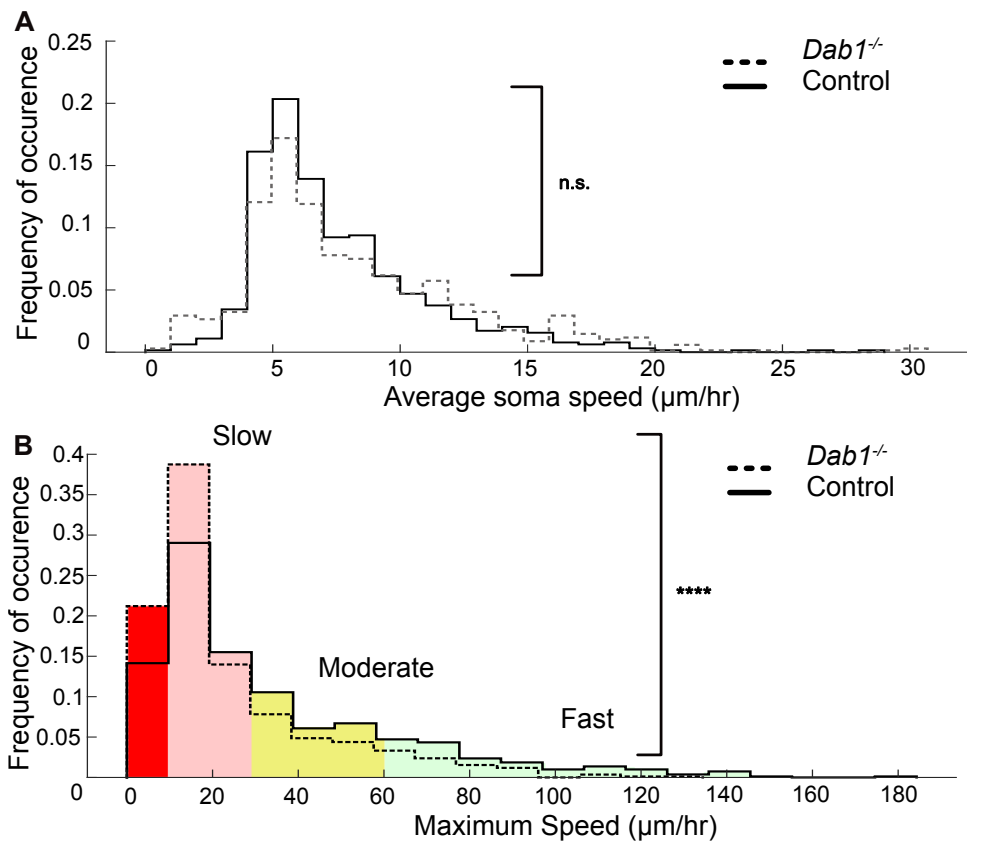

C

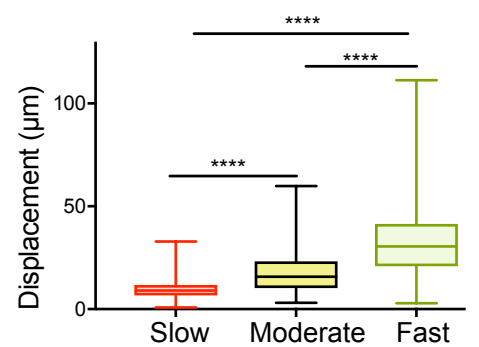

E

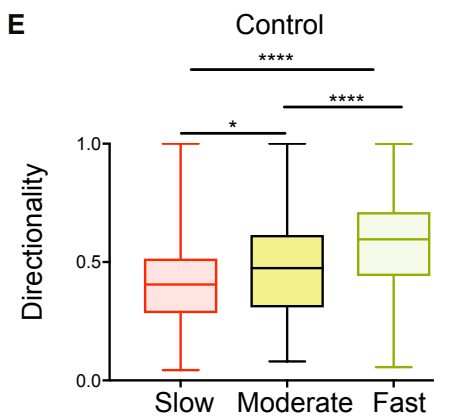

D

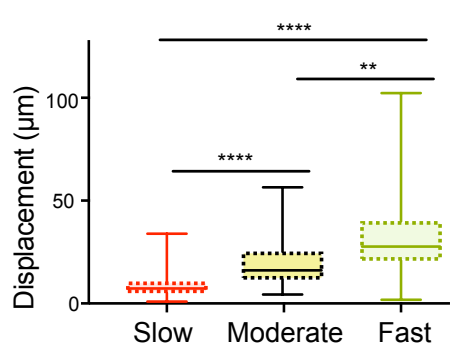

$\mathbf{F}$

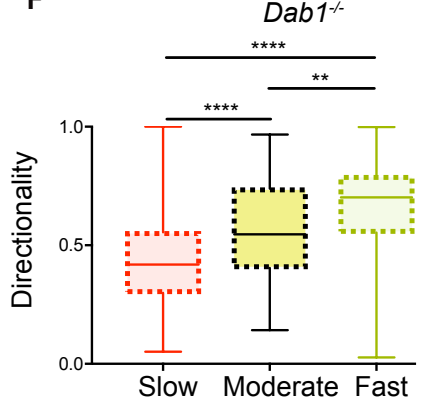

Figure 5. Reelin promotes infrequent, fast movements in mDA neurons. (A) Distribution of average soma speeds are not significantly altered in Dab1-/-slices ( $p=0.0657$, Mann-Whitney's test, $n=680$ control, 639 Dab1-/- mDA neurons). (B) Dab1-/- mDA neurons have significantly lower max-speeds compared to controls ( $* \star \star * ~ p<0.0001$, Mann-Whitney's test, $\mathrm{n}=806$ control, 844 Dab1-/- mDA neurons). Non-migratory (max-speed 0 - $10 \mu \mathrm{m} / \mathrm{hr}$ ), slow (10 - $30 \mu \mathrm{m} / \mathrm{hr})$, moderate (30 - $60 \mu \mathrm{m} / \mathrm{hr})$ and fast cells ( $>60 \mu \mathrm{m} / \mathrm{hr}$ ) are indicated by dark red, light red, yellow and light green colors respectively. (C,D) Total displacement (3D) of mDA neurons is significantly higher in moderate compared to slow mDA neurons, and highest in fast mDA neurons in both control (C) and Dab1-/-(D) brains. (E,F) Directionality (defined as ratio of total displacement to path length) in control and Dab1-/- slices is the least in slow, higher in moderate and the highest in fast mDA neurons. (C-F) * $p$ $<0.05, * * \mathrm{p}<0.01, * * * * \mathrm{p}<0.0001$, Kruskal-Wallis test; $\mathrm{n}=680$ control; $\mathrm{n}=639 \mathrm{Dab1}^{-/-}$cells, 3 slices/genotype.

Figure 5 -Figure supplement 1. Variation in instantaneous soma speed of mDA neurons.

Figure 5 -Figure supplement 2. Individual fast, moderate and slow mDA neurons from Dab1-/- slices have similar displacement and directionality profiles as control mDA neurons. 


\section{directed cell displacements}

We next asked whether max-speeds and directionality of migration were linked. We computed directionality as the ratio of total displacement (the 3D displacement between the initial and final positions of the neurons) to path length (the distance travelled by each neuron summed up irrespective of direction) Petrie et al. (2009) for migrating SN-mDA populations in control and Dab1-1- slices. A high value of directionality (maximum value $=1$ ) indicates almost no change in migratory direction while low values indicate frequent changes in direction. We found that directionality as well as total displacement generally increased with increasing max-speeds in $\mathrm{SN}$ $\mathrm{mDA}$ populations from both control and Dab1-/- slices (Figure 5C-F; Figure 5 - Figure supplement 2). These data indicate that the infrequent moderate-to-fast movements in SN-mDA neurons result in major contributions to the directed migration of these cells. Since Reelin signaling increases the fraction of SN-mDA neurons that are able to undergo moderate-to-fast movements, Reelin supports directed migration of mDA neurons on a population level.

\section{Reelin promotes preference for laterally-directed migration in mDA neurons}

As tangential migration ultimately results in SN-mDA migration away from the midline, we analyzed the trajectories of migratory SN-mDA neurons in the presence and absence of Reelin signaling. We determined the "trajectory angle" for each cell as the angle between the midline ( $y$-axis in live-images) and the cell's displacement vector (Figure 6A). Thus, a trajectory angle of $90^{\circ}$ indicates a cell whose total movement is precisely aligned to the lateral axis ( $x$-axis in live-images). We defined a cell as migrating laterally if its trajectory angle was between $45-135^{\circ}$. We then evaluated the angular mean and standard deviation $\left(\sigma_{\text {ang }}\right)$ for SN-mDA populations in control and Dab1-/- slices Berens (2009). We found that SN-mDA neurons from control slices displayed an anisotropy towards lateral migratory directions (mean $92.5^{\circ}, \sigma_{a n g} 68.4$ ) while Dab1-/- SN-mDA neurons showed a significantly reduced preference for lateral migration (mean $27.5^{\circ}, \sigma_{\text {ang }} 70.4$ ) (see materials and methods for analysis of circular variables)(Figure 6B-D).

Next, to evaluate if 'fast', 'moderate' and 'slow' cell populations of control and Dab1-/- slices showed differences in their preference for lateral migration, we analyzed their trajectories separately. We found that trajectories of all three SN-mDA groups were anisotropic in controls, favoring migration towards lateral directions, but this anisotropy was greater in 'fast' and 'moderate' cells than in 'slow' cells (Figure 7A,D,G). Resolving this further into individual slow, moderate and fast migratory phases in the migratory mDA population, we also found that individual moderate-to-fast phases were more anisotropic than slow phases (Figure 7- Figure supplement 1A,C,E).

In the absence of Reelin signaling, the trajectory profiles of 'slow' neurons were significantly altered with a complete loss of anisotropy towards lateral directions (mean $-12.3^{\circ}, \sigma_{\text {ang }} 69.7^{\circ}$ ) (Figure $7 \mathrm{~B}, \mathrm{C}$ ). In contrast, 'moderate' and 'fast' neurons still navigated to more lateral regions in Dab1-/slices and their trajectory angle distributions were nearly identical to control neurons (Dab1-/'moderate' neurons: mean $69.4^{\circ}, \sigma_{\text {ang }} 58.7^{\circ}$; 'fast' neurons: mean $81^{\circ}, \sigma$ ang $57.9^{\circ} ;$ ) (Figure 7D-I). This finding also applies to slow, moderate and fast phases: slow phases are weakly laterally directed in controls, but in the absence of Reelin signaling individual slow migratory movements lose their slight lateral preference (Figure 7- figure supplement 1B,D,F and data not shown). These results show that Reelin signaling promotes lateral migration of SN-mDA neurons by increasing the fraction of $\mathrm{SN}$-mDA neurons undergoing moderate-to-fast movements that are strongly biased for tangential movements and by promoting lateral anisotropy of 'slow' neurons.

mDA neurons adopt a bipolar morphology during moderate-to-fast phases of migration

Having thus defined the complex regulation of SN-mDA speed and trajectory profiles by Reelin signaling, we investigated the cellular morphology that underlies mDA tangential migration. Since the dynamic cell morphologies of migrating SN-mDA neurons have not been assessed previously, we first evaluated morphological changes in control SN-mDA neurons. Some cells had a stable, unbranched leading process (LP), and did not change their morphology, while other cells displayed dynamic LPs, that extended, retracted and branched frequently over time (Figure 8A-D; Figure 8 Figure supplement 1; Movie 2). 
A

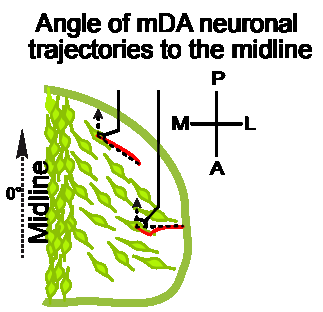

C

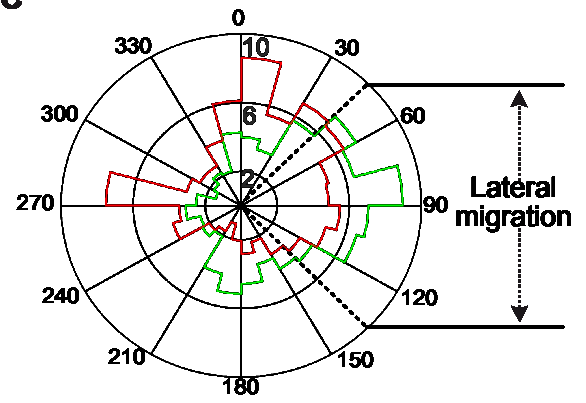

B

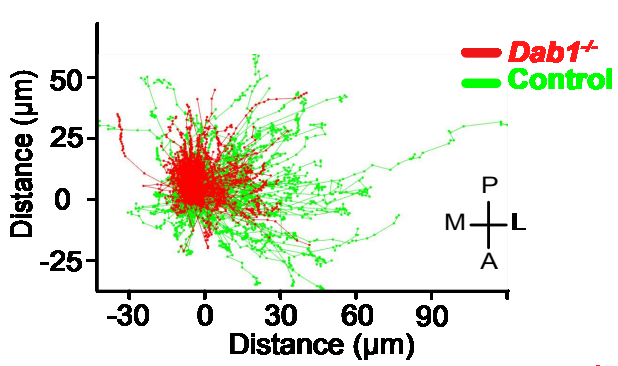

D
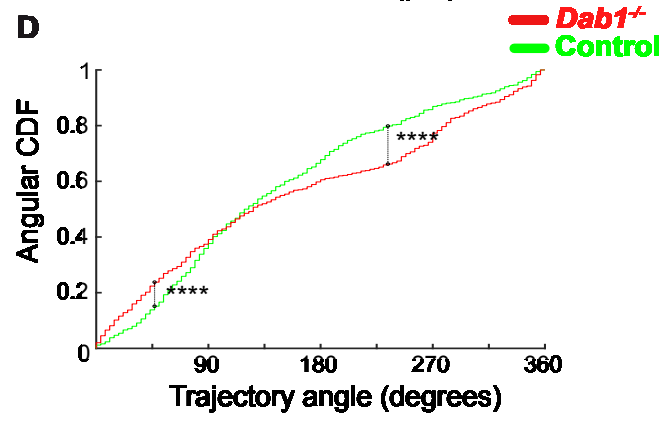

Figure 6. $m D A$ lateral migration is reduced in the absence of Reelin signaling $(A)$ Schematic showing trajectory angle measurement. (B) Trajectories of control (green) and Dab1-/- (red) mDA neurons from 1 control and $1 \mathrm{Dab} 1^{-/}$- slice imaged over the same duration), plotted relative to their starting point show loss of lateral directionality in Dab1-/- mDA neuron trajectories. (C) Polar histogram for angle of the mDA trajectories to the midline $\left(0^{\circ}\right)$ for all control (green) and Dab1-/- (red) mDA neurons analyzed. (D) Circular statistical analysis for angular distributions in (C) of control (green) and $\mathrm{Dab1}^{-/-}$(red) mDA neurons shows significant decrease in lateral anisotropy for Dab1-/-slices (**** $p<0.0001$, Kuiper's test for circular variables; $n=680$ control, $n=639$ Dab1-/- mDA neurons).

We studied the cell morphology of SN-mDA neurons ( 70 'fast', 40 'moderate' and 40 'slow' cells) in control and in Dab1-/- slices (49 'fast', 40 'moderate' and 40 'slow' cells) and examined whether slow, moderate and fast migratory phases were associated with specific morphologies (for details of morphological analysis see materials and methods). We defined three morphological categories: a neuron was considered to be 'bipolar-unbranched' when a maximum of two processes arose directly from the soma and the LP was unbranched. Bipolar cells that extended a branched LP were defined as 'bipolar-branched'. Neurons with more than two processes arising from the soma were defined as 'multipolar' (Figure 8A,C; Figure 8 - Figure supplement 1). The morphology of $\mathrm{SN}-\mathrm{mDA}$ neurons evaluated based on YFP expression was indistinguishable from their morphology as assessed by TH-immunostaining in cleared whole-mount brains at E13.5 (Movie 3).

To investigate whether specific morphologies observed in SN-mDA neurons were associated with specific migratory speeds, we broke down the morphology of these cells into time points during which they were in bipolar-unbranched, bipolar-branched or multipolar phases (Figure 8 A,C, Figure 8 - Figure supplement 1 ) and paired their morphology with soma speed (as calculated by change in soma position between the current and the subsequent time point). Bipolarity was predominant in all phases of migration, but in both control and $D a b 1^{-/-} \mathrm{SN}-\mathrm{mDA}$ neurons, fast and moderate migratory phases were almost exclusively associated with bipolar morphology. In contrast, about a third of slow migratory phases were associated with multipolar morphology. Hence, while slow migratory phases can occur in either bipolar or multipolar morphology, fast and moderate migration events are predominantly associated with bipolar morphology.

mDA neurons display unstable branch and leading process morphology in the absence 

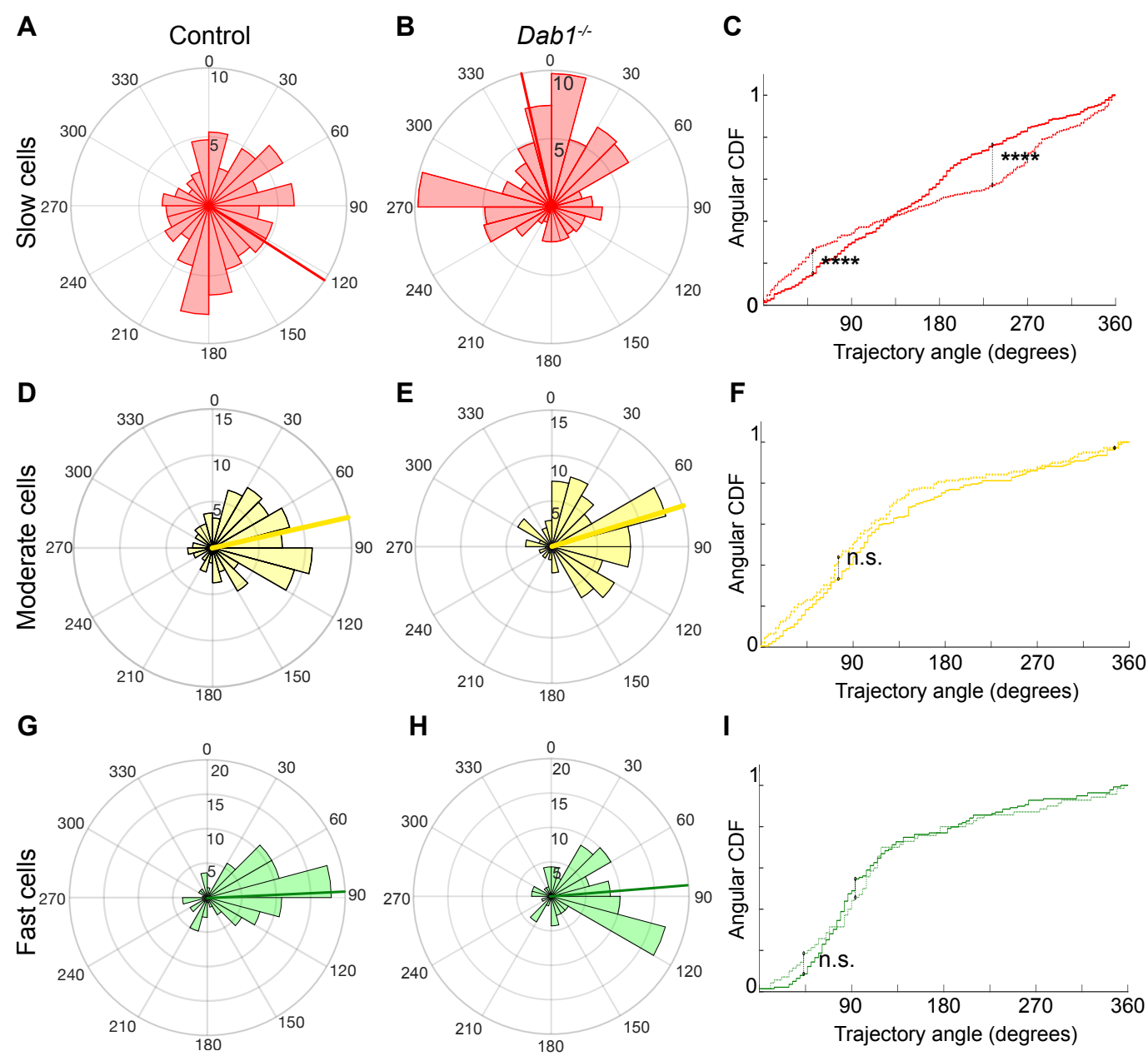

I

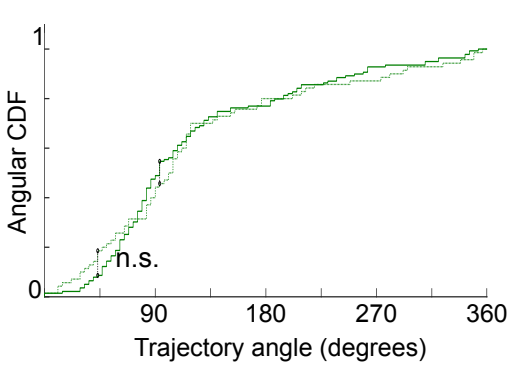

Figure 7. Reelin promotes preference for lateral migration in slow mDA neurons. $(A, B)$ Polar histogram for angle of slow mDA trajectories to the midline shows that slow cells have the least preference for lateral migratory direction in both control (A) and $\mathrm{Dab1}^{-/-}$(B) slices. (C) Circular statistical analysis for angular distributions of slow mDA neurons shows significant loss of preference for lateral migration in slow Dab1-/mDA neurons compared to controls (**** $p<0.0001$, Kuiper's test for circular variables; $n=355$ control, 480 Dab1-/-mDA neurons). (D-I) Moderate (D,E) and fast mDA neurons $(G, H)$ show high preference for lateral migration. Moderate (F) ( $\mathrm{n}=186$ control, $\mathrm{n}=139 \mathrm{Dab} 1^{-/-} \mathrm{mDA}$ neurons) and fast (I) $(\mathrm{n}=139$ control, $\mathrm{n}=70$ Dab1-/- mDA neurons) are laterally directed and their angular distributions are comparable in control and Dab1-/- slices. Red $(A, B)$, yellow $(D, E)$ and green $(G, H)$ lines represent mean angular direction for slow, moderate and fast populations, respectively. Figure 7 - Figure supplement 1. Lateral migration occurs during moderate and fast migratory phases of mDA neurons.

\section{of Reelin signaling}

In time-lapse data-sets, some mDA neurons transitioned between bipolar and multipolar morphology, while others maintained either a bipolar or multipolar morphology during imaging. We next examined the proportions of migrating SN-mDA neurons that displayed a constant bipolar (branched and unbranched), constant multipolar or transitionary morphology over time (Figure $8 \mathrm{~A}, \mathrm{C}$; Figure 8 - Figure supplement 1 ). This analysis enabled us to ask whether morphological stability is altered in the absence of Reelin signaling. In controls, transitionary cells made up about $40 \%$ of the total population. The proportion of transitionary cells was significantly increased in the Dab1/1- population, while the population of bipolar neurons was decreased (Figure 9A, Table 1 ). Within the transitionary population, we found however no difference in the frequency of transitions between bipolar and multipolar morphologies for each neuron (defined as number of morphology 
A

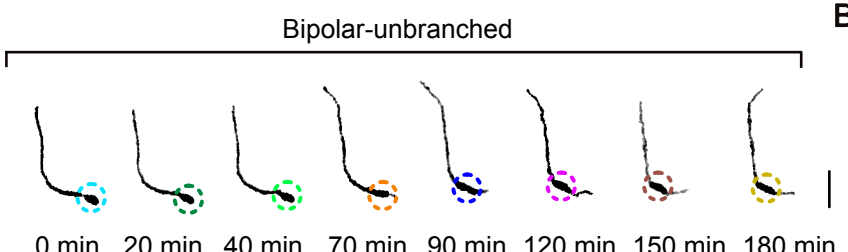

C

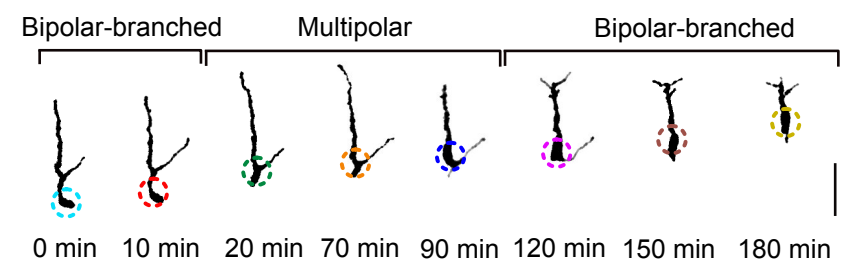

B
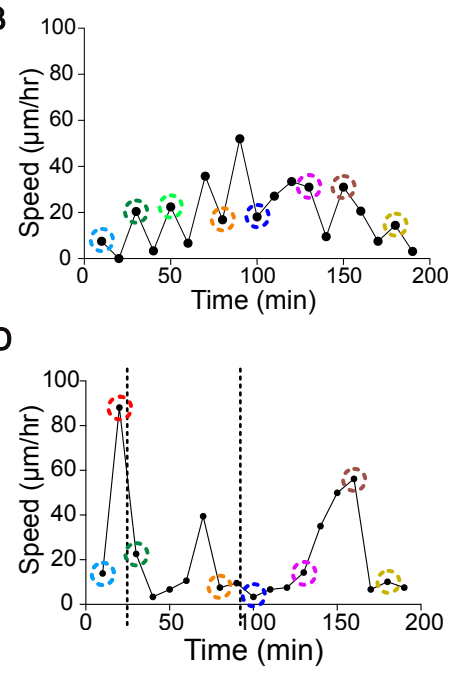

$\mathrm{E}$

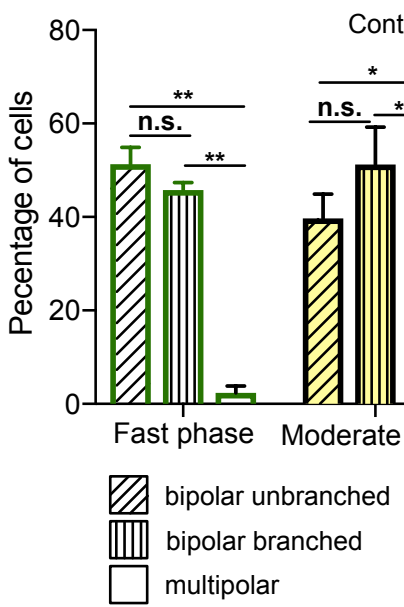

$\mathrm{F}$

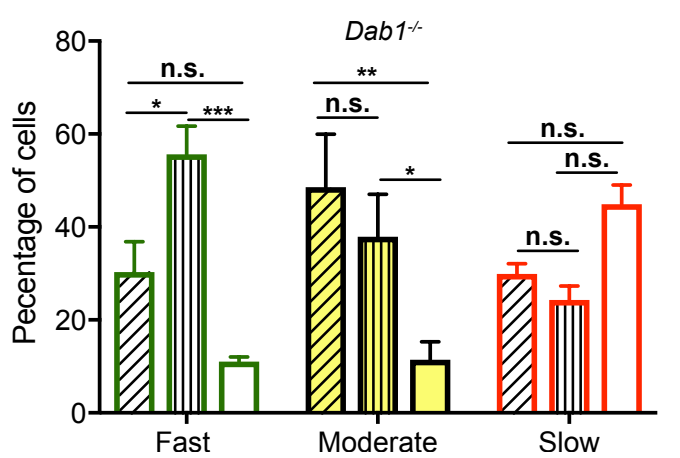

Figure 8. Moderate-to-fast migratory phases are strongly associated with bipolar morphologies in mDA neurons. (A) Control mDA neuron displaying a 'bipolar-unbranched' morphology at all analyzed time-points. (B) Soma speed profile of mDA neuron shown in (A). (C) Control mDA neuron transitioning between bipolar and multipolar morphology. At $t=0 \mathrm{~min}$, the cell has a branched leading process (LP), the cell soma moves along the LP to reach the branch-point and takes up a multipolar morphology ( $t=20 \mathrm{~min})$. The cell remains multipolar until $t=90 \mathrm{~min}$, after which one process is retracted $(\mathrm{t}=120 \mathrm{~min})$ and the cell resumes a bipolar morphology ( $t=150 \mathrm{~min}$ ). Bipolar phase: one or two processes arise directly from the soma. Multipolar phase: more than two processes arise directly from the soma. $(A, C)$ Colored circles: soma as defined by the tracking process. Scale bar: $25 \mu \mathrm{m}$. (D) Soma speed for the neuron in (C) is higher during its bipolar phase. $(\mathrm{E}, \mathrm{F})$ Proportion of bipolar unbranched and branched morphology is significantly higher during fast and moderate phases of migration, while slow phase shows higher proportion of multipolar cells in both control (E) and Dab1-/- (F) mDA neurons ( ${ }^{*} p<0.05$, $* * p<0.005, * * * p<0.001, * * * * p<0.0001$; two-way ANOVA). Figure 8 Figure supplement 1. Morphological characterization of mDA neurons

transitions per hour) in Dab1-/- compared to control slices (Figure 9). We then examined the appearance and disappearance of processes both on the soma and the LP of transitionary neurons ( $\mathrm{n}=64$ in control, $\mathrm{n}=70$ in $\mathrm{Dab1^{-/- }}$ ) in further detail (Figure 9C; Figure 9 - Figure supplement 1). We found that these branch transition events were significantly more frequent in Dab1-/- SN-mDA transitionary neurons (Figure 9D), since Dab1-/- neurons displayed short, transient protrusions that appeared on the soma and LP for only a few time frames before disappearing (Figure 9 - Figure supplement 1).

Finally, we randomly selected 20 control and 20 Dab1-/- mDA neurons with maximum soma speed of more than $10 \mu \mathrm{m} / \mathrm{hr}$ and manually traced their morphology in 3D for the first 19 imaging time-points (Figure 9 - Figure supplement 2). In all control and Dab1-/- mDA neurons, the LP 
remained stable and visible during the duration of imaging. We then compared the length of the

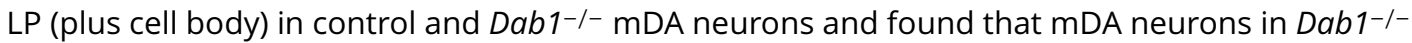
slices displayed a broader distribution of LP length with very long and very short LPS (Figure 9 Figure supplement 2G). Hence, in the absence of Reelin signaling, SN-mDA neurons display aberrant changes in morphology characterized by an increased proportion of transitionary neurons, an increase in unstable processes on the cell soma and LP and a greater variation in LP length.

Table 1. Morphology of mDA neurons in control and Dab1-/- slices

\begin{tabular}{|c|c|c|c|c|c|c|c|}
\hline \multirow[b]{2}{*}{ Cell type } & \multirow[b]{2}{*}{ Morphology } & \multicolumn{3}{|c|}{ Control } & \multicolumn{3}{|c|}{ Dab1-/- } \\
\hline & & Slice 1 & Slice 2 & Slice 3 & Slice 1 & Slice 2 & Slice 3 \\
\hline \multirow{3}{*}{ Fast } & Bipolar & $15 / 27$ & $12 / 26$ & $7 / 17$ & $5 / 16$ & $6 / 15$ & $5 / 18$ \\
\hline & Transitionary & $12 / 27$ & $13 / 26$ & $10 / 17$ & $9 / 16$ & $8 / 15$ & $12 / 18$ \\
\hline & Multipolar & $0 / 27$ & $1 / 26$ & $0 / 17$ & $2 / 16$ & $2 / 15$ & $1 / 18$ \\
\hline \multirow{3}{*}{ Moderate } & Bipolar & $8 / 16$ & $4 / 10$ & $4 / 14$ & $4 / 13$ & $5 / 19$ & $5 / 8$ \\
\hline & Transitionary & $7 / 16$ & $6 / 10$ & $10 / 14$ & $9 / 13$ & $14 / 19$ & $3 / 8$ \\
\hline & Multipolar & $1 / 16$ & $0 / 10$ & $0 / 14$ & $0 / 13$ & $0 / 19$ & $0 / 8$ \\
\hline \multirow{3}{*}{ Slow } & Bipolar & $3 / 11$ & $7 / 12$ & $7 / 17$ & $5 / 14$ & $5 / 15$ & $2 / 11$ \\
\hline & Transitionary & $2 / 11$ & $1 / 12$ & $3 / 17$ & $5 / 14$ & $5 / 15$ & $5 / 11$ \\
\hline & Multipolar & $6 / 11$ & $4 / 12$ & $7 / 17$ & $4 / 14$ & $5 / 15$ & $4 / 11$ \\
\hline
\end{tabular}

\section{Reelin downstream signaling in the ventral midbrain}

As it is not known which downstream components of the Reelin signaling pathway regulate $\mathrm{SN}$-mDA tangential migration, we investigated Reelin signaling events that were previously shown to influence neuronal polarity in migrating neurons in the cortex, hippocampus or spinal cord. Reelin signaling results in the activation (phosphorylation) of PI3K (Phosphatidylinositol-4,5-bisphosphate 3-kinase) through DAB1 Jossin and Goffinet (2007). PI3K activation results in phosphorylation (activation) of LIMK1 (Lim domain kinase 1) via Rac1/Cdc42 and PAK1. P-LIMK1 inactivates (phosphorylates) Cofilin1, an actin depolymerizing protein of the ADF/Cofilin family. Reelin-mediated inactivation of Cofilin 1 ultimately leads to the stabilization of the actin cytoskeleton and has been implicated in stabilizing LPs of radially migrating cortical neurons as well as in preventing the aberrant tangential migration of neurons of the autonomous nervous system in the spinal cord Maciver and Hussey (2002), Krüger et al. (2010), Franco et al. (2011), Chai et al. (2009); Frotscher et al. (2017). To detect a potential misregulation of these downstream events in absence of Reelin signaling, we performed immunoblotting on E14.5 embryonic ventral midbrain tissue for $p$-LIMK1/LIMK1 and $\mathrm{p}$-Cofilin1/Cofilin1. We did not detect significant differences in protein levels or in relative phosphorylation levels (Figure 10 and data not shown). Hence, we conclude that the regulation of LIMK1/Cofilin1 activity is unlikely to be the key event in controlling cytoskeletal stability in migrating mDA neurons downstream of Reelin signaling.

Next, we examined Cadherin2 ( $\mathrm{CDH} 2)$ expression in the ventral midbrain. Reelin signaling controls somal translocation of radially migrating cortical neurons by modulating cell adhesion properties through regulation of $\mathrm{CDH} 2$ via the Crk/C3G/Rap1 pathway Franco et al. (2011). Relative protein levels of $\mathrm{CDH} 2$ were similar in tissue lysates from control and Dab1-/- E14.5 ventral midbrain (Figure 10 - Figure supplement 1 ). Whether $\mathrm{CDH} 2$ levels are altered at the membrane of mDA neurons in $\mathrm{Dab1}^{-/-}$mice could not be assessed, since the immunostaining for $\mathrm{CDH} 2$ on sections was not of sufficient quality to make a clear assessment of changes in membrane localization. 
A

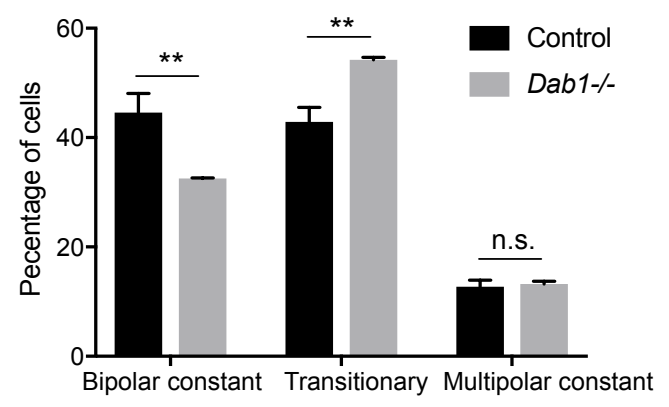

B

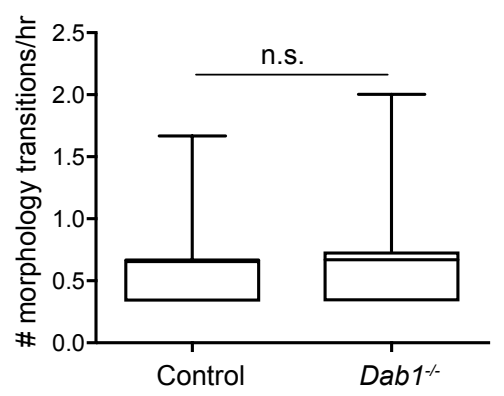

C

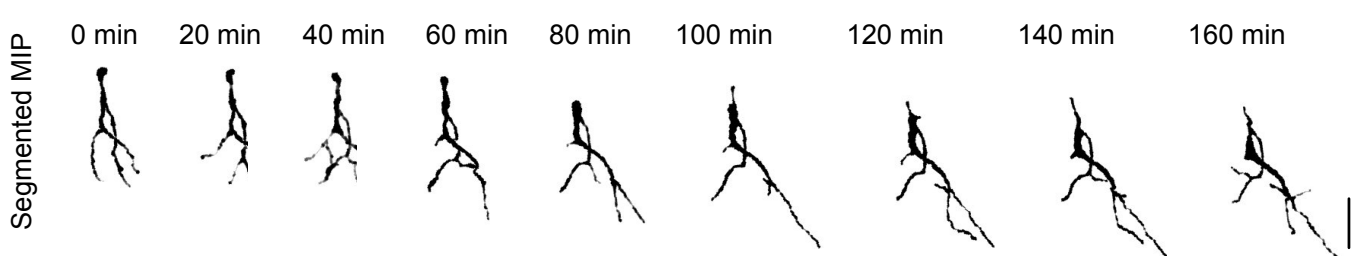

D

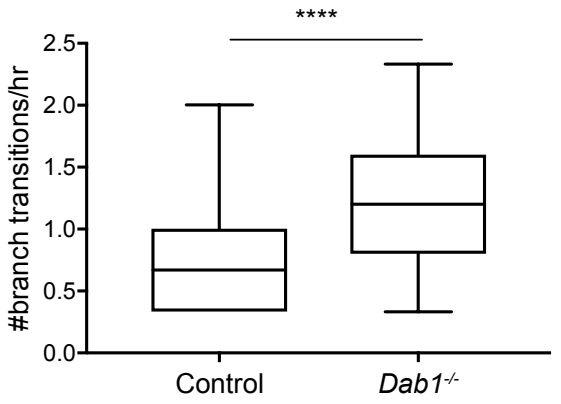

Figure 9. Decreased stability of neuronal morphology in mDA neurons. (A) Relative proportion of constantly bipolar mDA neurons are decreased, while transitionary mDA neurons are increased in Dab1/-/-slices. (B) Frequency of transitions from multipolar to bipolar phase (and vice versa) are not significantly altered in the absence of Reelin signaling ( $p=0.6922$; Mann-Whitney's test). (C) Bipolar and multipolar phase of a Dab1-/-transitionary mDA neuron. In this example, the bipolar phase lasts from $t=0$ min to $t=60 \mathrm{~min}$. In the multipolar phase (starting at $\mathrm{t}=80 \mathrm{~min}$ ) many, unstable protrusions form. Scale bar: $25 \mu \mathrm{m}$. See Figure 9-

Figure supplement 1 for more detail. (D) Quantification of appearance and disappearance of branches (defined as branch transitions per hour) in control and Dab1-/- mDA transitionary neurons shows a significant increase in branch transitions in mDA neurons in Dab1-/- slices(**** $p<0.0001$; Mann-Whitney's test). Figure 9 - Figure supplement 1. Absence of Reelin signaling results in the formation of unstable protrusions on the soma and leading process of mDA neurons.

Figure 9 - Figure supplement 2. Greater spread in length of leading process in Dab1-/-mDA neurons.

\section{Discussion}

The correct tangential migration of mDA neurons is crucial for the formation of the SN. Our study provides the first comprehensive insight into speed, trajectory and morphology profiles of tangentially migrating mDA neurons, and uncovers the alterations of tangential migratory behavior that result in aberrant SN formation in the absence of Reelin signaling (Figure 11).

\section{Reelin signaling directly regulates tangential migration of SN-mDA neurons}

A number of previous studies established the importance of Reelin in the formation of the SN Kang et al. (2010), Nishikawa et al. (2003), Sharaf et al. (2013), Bodea et al. (2014), but it remained to be elucidated whether Reelin is directly required for the tangential migration of SN-mDA neurons. Studies in cortex have shown that while Reelin is directly required for the stabilization of the LP and for the orientation of radially-migrating cortical projection neurons Franco et al. (2011), Reelin also indirectly affects migration through regulating radial glia cell process extension, morphol- 
A

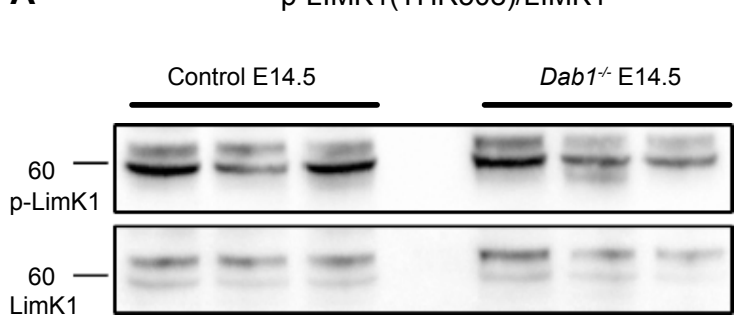

C

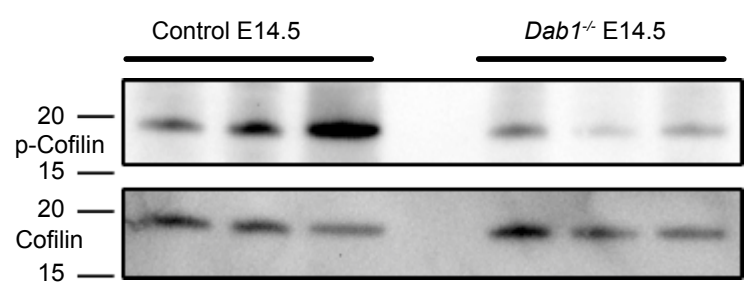

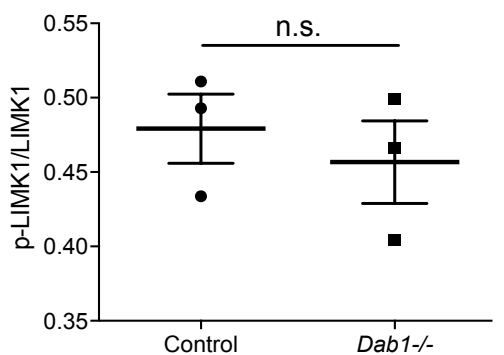

D

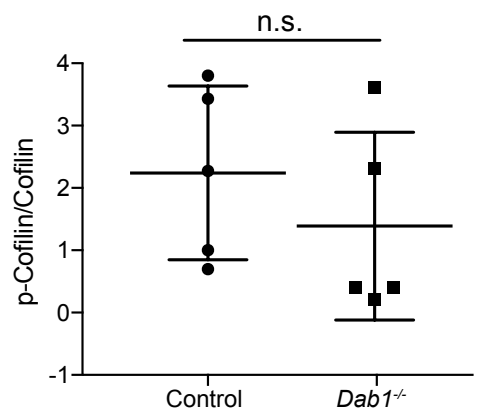

ogy and maturation Hartfuss et al. (2003), Keilani and Sugaya (2008). Tangentially migrating cortical interneurons are only indirectly affected by Reelin signaling: the improper cortical layering caused by defective radial migration in absence of Reelin signaling ultimately results in incorrect positioning of interneurons Yabut et al. (2007). Reelin also plays a role in interneuron precursors that undergo tangential chain migration to the olfactory bulb. However, it does not modulate tangential migration directly but rather acts as a detachment signal that regulates the switch form tangential chain migration to radial migration Hack et al. (2002). Evidence for a direct function of Reelin signaling in tangential neuronal migration comes from sympathetic preganglionic neurons in the spinal cord. In these neurons, Reelin has been shown to stabilize LPs via the phosphorylation of Cofilin 1 during tangential migration thereby preventing aberrant migration Krüger et al. (2010), Phelps (2002).

To explore whether Reelin has a direct role in tangential migration of SN-mDA neurons, we inactivated Dab1 in SN-mDA neurons starting at the onset of their tangential migration without affecting their earlier radial migration step and without inactivating Dab1 in other cell populations in the ventral midbrain. The similarity in mediolateral distribution of SN-mDA neurons in Dab1//and in Dab1 CKO implies that Reelin signaling has a direct effect on migrating SN-mDA neurons. We also confirmed that the GIRK2-expressing mDA population, which consists of lateral VTA- and SN-mDA neurons was distributed in a similar manner than what we reported previously for $\mathrm{Dab}^{-1-}$ mice Bodea et al. (2014). Investigation of additional markers that label SN-mDA neurons more specifically, such as $L m o 3$ and SOX6, showed that the medially misplaced SN-mDA neurons were partially intermingled with VTA-mDA neurons. These results imply that in absence of Reelin signaling in mDA neurons, the separation of SN- and VTA-mDA neurons is not fully completed and SN-mDA neurons lose their ability to undergo the long-range tangential migration necessary to form the laterally-positioned SN. Thus, our findings are the first demonstration of Reelin as a direct regulator 
of tangential neuronal migration in the brain.

\section{Reelin protein is localized in the lateral ventral midbrain}

In the ventral midbrain, Reelin mRNA is restricted to the cells of the red nucleus at E13.5 and E14.5 Bodea et al. (2014) (Figure 2). Using immunostaining, we show that Reelin protein is distributed much more broadly at these stages. Strong labeling is seen in regions lateral to the migrating SN-mDA, while weaker staining is observed in the area where SN-mDA neurons are localized. Thus, the Reelin protein distribution that we describe here is consistent with a direct role of Reelin in regulating SN-mDA migration. Whether the red nucleus is the only source for Reelin in the ventral midbrain or whether there are additional sources remains to be investigated. Mouse mutants in which the RN is only partially formed do not show any obvious displacement of SN-mDA neurons (at least not up to E18.5), suggesting that other Reelin sources could be important for mDA migration Prakash et al. (2009). Reelin mRNA is expressed anterior to the SN, in the hypothalamus and ventral thalamus Alcántara et al. (1998). Moreover, it has been proposed that Reelin is transported from the striatum to the SN via axons in the striatonigral pathway Nishikawa et al. (2003). Indeed, Reelin is expressed in the early differentiating cells in the striatum, but the striatonigral pathway is probably only established (E17 in rat) after the critical time period for SN-mDA migration Fishell and Kooy (1987), Alcántara et al. (1998).

\section{Reelin promotes the proportion of mDA neurons undergoing fast, directed migration}

The visualization and tracking of a large population of migrating mDA neurons, and the subsequent categorization of the instantaneous soma speed of individual mDA neurons into slow, moderate and fast phases revealed that irrespective of their max-speed, mDA neurons spent a majority of their time at rest. During their migratory phase, mDA neurons move mostly at slow speed. Moderate-to-fast laterally-directed migration spurts that result in large displacements are infrequent and occur in only a subset of labeled mDA neurons during the time-window of imaging. Thus, mDA neurons migrate in two modes: in a frequent, slow mode and in infrequent, fast movements with a strong lateral orientation. A similar pattern of migration with variable instantaneous speeds and periods of rest has also been reported for newly generated granule cells in the dentate gyrus and for cortical projection neurons Simó et al. (2010), Wang et al. (2018).

Comparing mDA tangential migration in the presence and absence of Reelin signaling, we observed that the duration of the individual migratory phases as well as average speed distribution

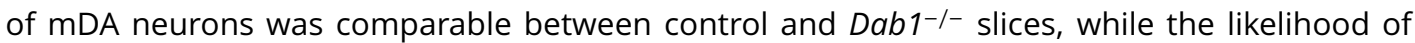
moderate-to-fast migration events was decreased in mDA neurons in Dab1-/- slices. In addition, a higher proportion of mDA neurons spent the entire imaging period at rest. Hence, Reelin promotes the likelihood with which moderate-to-fast migration spurts occur and increases the proportion of cells that enter a migratory phase.

Interestingly, the increased presence of activated DAB1 in cortical projection neurons as a consequence of reduced ubiquitylation and degradation in absence of the E3 ubiquitin Ligase Cullin-5 leads to the opposite effect in the migratory behavior of these neurons: periods of rest are decreased and average as well as instantaneous speed is increased at late stages of cortical migration (E16.5) Simó et al. (2010). This would be consistent with the role of Reelin that we observe in the migration of mDA neurons. In contrast, average speed appears not to be altered in cortical neurons of reeler mutants at this stage of development Chai et al. (2016). Observation of cortical projection neurons in their multipolar-to-bipolar transition phase at E15.5 suggests yet another effect of Reelin: at this stage cortical neurons were observed to migrate faster in the absence of Reelin signaling while addition of exogenous Reelin slowed down migrating neurons, but only within the subventricular zone Britto et al. (2011), Britto et al. (2013). Thus, even in the same neuronal population, Reelin signaling might have diverse effects on the speed of neuronal migration at different stages of migration.

\section{Reelin promotes a preference for directed migration}

While moderate-to-fast migratory events are less likely in the Dab1-/- mDA population, individual

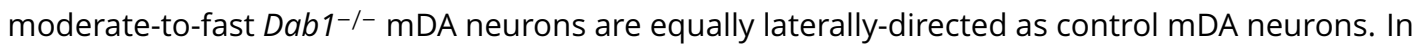


contrast, slow cells, which are weakly anisotropic in controls are significantly more isotropic in Dab1-/- slices. The loss of the laterally-directed slow movements might interfere with mDA neuron's ability to initiate moderate-to-fast, laterally-directed spurts. Indeed, mDA neurons have an aberrant orientation in E13.5 reeler brains Bodea et al. (2014). In the cortex, Reelin regulates orientation and cell polarity of multipolar neurons in the intermediate zone facilitating their switch to bipolar, glia-dependent migration Gärtner et al. (2012), Gil-Sanz et al. (2013), Jossin and Cooper (2011). Cortical projection neurons in their early phase of migration have been shown to deviate from radial migratory trajectories, in the absence of Reelin signaling as well as in the presence of exogenous Reelin Britto et al. (2011), Britto et al. (2013), Chai et al. (2016). Reelin also promotes directionality during the radial migration of dentate gyrus cells Wang et al. (2018). Interestingly, a recent study provides evidence that mDA neurons derived from induced pluripotent stem cells homozygous or heterozygous for a REELIN deletion show a disruption in their directed migratory behavior in neurosphere assays. Since the disruption occurs in absence of any organized tissue structure, Reelin signaling seems to modulate the ability of mDA neurons for directed migration independently of a specific pattern of Reelin protein deposition in the surrounding tissue Arioka et al. (2018). In conclusion, Reelin appears to be a crucial factor in enabling SN-mDA neurons to initiate directed migration rather than a factor that guides SN-mDA neurons in a particular direction.

\section{Reelin signaling promotes stable morphologies in SN-mDA neurons}

We show that moderate and fast movements of mDA neurons are strongly associated with bipolar morphologies both in control and Dab1-/- slices. Bipolarity is still predominant in slow phases, but about a third of the slow phases are associated with a multipolar morphology. In control slices, more than half of mDA neurons maintain a bipolar morphology throughout the imaging period, while about $40 \%$ transition between multipolar and bipolar morphologies. Only a small subset of cells (about 10\%) stays multipolar at all time points. In absence of Reelin signaling, the percentage of transitionary cells is significantly increased, and the proportion of stable bipolar cells is decreased.

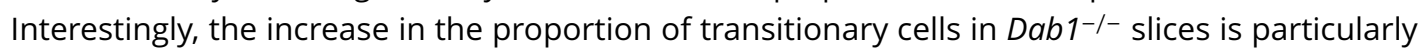
pronounced in the cell population that does not reach moderate-to-fast migration speeds and that is significantly more isotropic (data not shown) suggesting a correlation between loss of anisotropy in these cells and increased transitioning between bipolar and multipolar morphology. In transitionary cells of $D a b 1^{-/-}$slices, there is a significant increase in branch transitions at the soma and LP, a sign of decreased branch stability. Moreover, the length of the LP is significantly more variable in $\mathrm{Dab} 1^{-/-}$than in control neurons. Thus, Reelin signaling appears to promote stability of morphologies once they have been adopted at specific phases of migration in mDA neurons.

In cortical neurons, Reelin appears to have multiple effects on cell morphology. In dissociated cortical neuronal cultures, Reelin signaling results in an increase in filopodia formation, likely via activation of Cdc42 Leemhuis et al. (2010).Moreover, in presence ofexogenous Reelin in organotypic slice cultures, projection neurons in the ventricular zone display a greater proportion of multipolar morphology, a phenotype concomitant with reduced migratory speeds (see above, Britto et al. (2013)). In contrast, LP morphology of migrating cortical neurons is comparable in presence and absence of Reelin signaling when these neurons first contact the marginal zone of the cortex, but Reelin signaling is required to maintain this morphology and a stable LP during the final somal translocation step of these neurons Chai et al. (2016), Franco et al. (2011). Finally, a recent study showing the phosphorylation of DAB1 via the Netrin receptor deleted in colorectal cancer (DCC) has reported an increase in multipolar neurons in the subventricular zone of Dcc knockout cortex Zhang et al. (2018). In summary, depending on location, concentration, and sub-cellular localization, Reelin and DAB1 can have differing effects on the morphology of migrating neurons.

An indirect regulation of morphology by Reelin signaling has been reported in tangentiallymigrating cortical interneurons. In interneurons, branching of LPs aids in precise sensing of the extracellular environment during chemotaxis Martini et al. (2008). In the inverted reeler cortex, interneurons display a significantly higher number of branch nodes and higher length of LPs than interneurons in control brains Yabut et al. (2007). This aberrant morphology is accompanied by 
their ectopic location in cortical layers. Since interneurons do not directly require Reelin signaling for their migration, it is likely that their aberrant morphology in the reeler cortex is an indirect effect of their altered position. As we observe similar effects on cell morphology in Dab1 $1^{-/-}$mDA neurons, the aberrant mDA neuronal morphology may be a consequence of an increased necessity to scan the environment for guidance cues in ectopic medial positions rather than a direct downstream effect of Reelin.

\section{Reelin downstream signaling in SN-mDA neurons}

It has previously been demonstrated that the regulation of $\mathrm{CDH} 2$ via the Crk/CrkL-C3G-Rap1 pathway at the cell surface is important for the effect of Reelin on the polarity of cortical projection neurons during their migration Franco et al. (2011), Park and Curran (2008), Sekine et al. (2012), Voss et al. (2008). Cofilin1 has been shown to stabilize the LPs of migrating cortical neurons downstream of Reelin signaling-activated LIMK1 Chai et al. (2016), Chai et al. (2009). However, we demonstrate here that expression and/or phosphorylation levels of these Reelin downstream effectors are not obviously altered in mDA neurons in the absence of Reelin signaling. Other signaling events that influence cortical migration downstream or in parallel to Reelin signaling are mediated through integrin a5ß1 or the Netrin1-DCC pathway. The knockdown of integrin a5ß1 in cortical neurons affects apical process stability during terminal translocation suggesting that additional adhesion molecules may be recruited by Reelin signaling Sekine et al. (2012). In the cortex, both $\mathrm{CDH} 2$ and integrin a5ß1 act downstream of Reelin, with integrin a5ß1 anchoring the leading tip of terminally translocating neurons in the marginal zone and $\mathrm{CDH} 2$ regulating the subsequent cell movements Sekine et al. (2014). Interestingly, integrin a5ß1 has been shown to be important for stabilizing neurite extensions of mDA neurons in vitro. Whether it plays a general role in stabilizing neuronal processes in mDA neurons, including LPs, and in mDA migration has not been explored Izumi et al. (2017). Recently, cross talk between Netrin1-DCC and Reelin-Dab1 pathways has been reported in migration of cortical projection neurons Zhang et al. (2018). The Netrin1-DCC pathway is also important for proper localization of SN-mDA neurons during development Li et al. (2014), Xu et al. (2010). Though the effect on mDA distribution induced by Dcc inactivation differs from the effect caused by Dab1 inactivation, it is possible that effectors downstream of the Netrin1-DCC pathway, such as focal adhesion kinase my play a role in mediating Reelin signal in mDA neurons Zhang et al. (2018).

\section{Conclusion}

Here we provide a detailed characterization of the migratory modes and cellular morphologies underlying SN-mDA tangential migration to gain a detailed understanding of SN formation. We demonstrate that Reelin directly regulates lateral, tangential migration of MDA neurons by stabilizing the morphology of mDA neurons, by promoting lateral anisotropy in small, slow movements and by increasing the frequency of laterally-directed moderate-to-fast migration events that cover larger distances. We thus present new mechanistic insight into how Reelin signaling controls tangential migration and regulates the formation of the $\mathrm{SN}$ and open the door to further investigations of the molecular mechanisms of mDA migration.

\section{Methods and Materials \\ Mouse lines}

$D a b 1^{\text {flox }}$ and Dab1 ${ }^{\text {del }}$ mice Franco et al. (2011) were obtained from Dr. Amparo Acker-Palmer, University of Frankfurt. Dab1 CKO mice (genotype: $D A T^{\mathrm{Cre} /+}$, Dab1 ${ }^{\text {flox/del }}$ ) were generated by

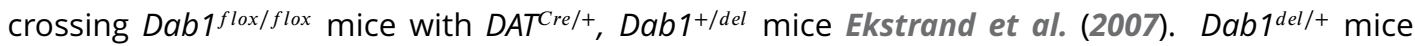
were used to generate complete knockouts of Dab1 (Dab1-/-). DATCre/+ mice were crossed with ROSAloxP-STOP-loxP-EYFP mice (R26 EYFP) Srinivas et al. (2001) to analyse the timing and extent of recombination. Mosaic labelling of migrating mDA neurons was achieved by crossing Shh ${ }^{\text {CreER }}$ mice Harfe et al. (2004) with R26 EYFP mice. Day of vaginal plug was recorded as E0.5. Mice were housed in a controlled environment, with 12-hour light/night cycles and ad libidum availability of 


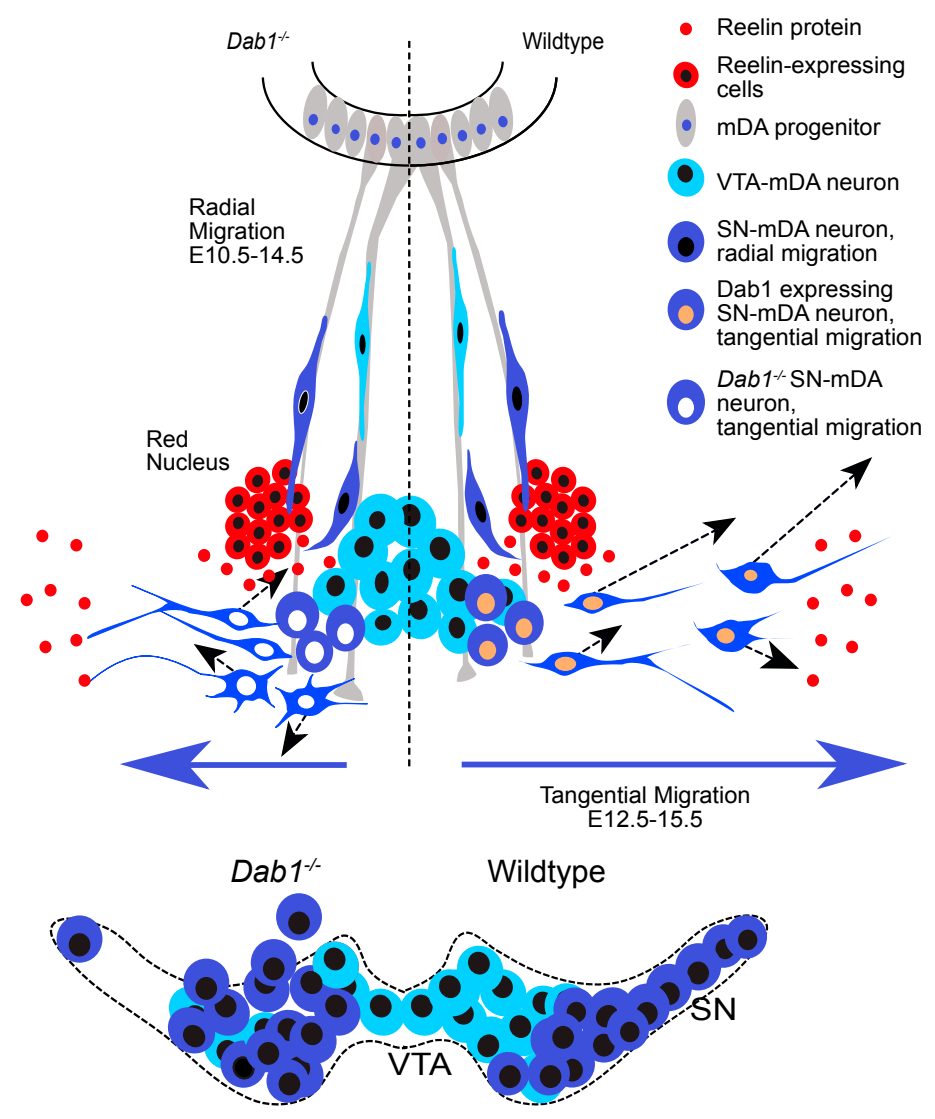

Figure 11. Schematic representation of Reelin regulation in mDA tangential migration. Reelin directly regulates lateral, tangential migration of mDA neurons and promotes fast, laterally directed mode of migration by regulating small lateral movements and stabilizing morphology of mDA neurons. In absence of Reelin signaling, slow movements in mDA neurons lose their lateral anisotropy, morphologies are less stable during migration and the fraction of neurons undergoing fast, laterally directed migration is reduced. This results in a medial clustering of SN-mDA neurons at late embryonic and postnatal stages.

food and water. All experiments were performed in compliance with the guidelines for the welfare of animals issued by the Federal Government of Germany and the guidelines of the University of Bonn.

\section{Tamoxifen}

Tamoxifen ( $75 \mathrm{mg} / \mathrm{kg}$ body weight) was administered by gavage to pregnant dams at E8.5 to label SN-mDA neurons Bodea et al. (2014). TM (Sigma Aldrich) was prepared as a $20 \mathrm{mg} / \mathrm{mL}$ solution in corn oil (Sigma Aldrich), with addition of progesterone (Sigma Aldrich, $5 \mathrm{mg} / \mathrm{mL}$ ) to reduce miscarriages.

\section{Immunohistochemistry}

Pregnant dams were sacrificed by cervical dislocation. Embryos were dissected in ice cold PBS. Heads (E13.5 - E15.5) or brains (E16.5 - E18.5) were fixed in 4\% paraformaldehyde (PFA) for 2 - 3 hrs at room temperature (RT). Adult mice were anesthetized with isofluorane, perfused transcardially with phosphate buffered saline (PBS), followed by $4 \%$ PFA. Tissue was cryopreserved in OCT Tissue Tek (Sakura), embryonic tissue was cryosectioned at $14 \mu \mathrm{m}$, adult brains were cryosectioned at $40 \mu \mathrm{m}$ thickness. Immunostaining was essentially performed as previously described Blaess et al. (2011).

For immunostainings, sections were fixed briefly in 4\%PFA (5 min at RT), followed by $1 \mathrm{hr}$ incubation in $10 \%$ NDS in $0.1 \%$ Triton in PBS (0.1\% PBT). Sections were incubated overnight at $4^{\circ} \mathrm{C}$ in primary antibody in 3\% NDS in $0.1 \%$ PBT. Sections were washed $3 \mathrm{X}$ in $0.1 \%$-PBT and incubated for 2 $\mathrm{h}$ in secondary antibody in 3\% NDS in $0.1 \%$ PBT before mounting with Aqua Polymount (Polysciences 
Inc.).

For the detection of SOX6, antigen retrieval was carried out in $0.1 \mathrm{M}$ EDTA for $30 \mathrm{~min}$ at $65^{\circ} \mathrm{C}$ before blocking, and Cy3-Streptavidin amplification was used with biotinylated donkey anti-rabbit antibody. To improve detection of DAB1 with rabbit anti-DAB1 antibody in E15.5 embryonic sections, a tyramide signal amplification (TSA) was carried out with the TSA kit (Perkin Elmer) as follows: Sections were blocked in the TSA kit blocking solution for $1 \mathrm{~h}$ followed by incubation with rabbit anti-DAB1 antibody (1:5000, Howell et al. (1997)) in 0.1\% TBST (Tris buffered saline with $0.1 \%$ Triton) overnight at $4^{\circ} \mathrm{C}$. After a washing step in TBST, sections were incubated for $2 \mathrm{~h}$ at RT with biotinylated donkey anti-rabbit in TBST, followed by another washing step and incubation with HRP conjugated Streptavidin (1: 1000) in TBST for $1 \mathrm{~h}$ at RT. Sections were again washed with TBST and incubated for 10 min with TSA detection reagent. After additional washing steps in TBST and $0.1 \%$ PBT sections were co-stained for TH following the standard immunostaining protocol. A complete list of primary and secondary antibodies is presented in Table 2.

\section{Immuno blotting}

WT and Dab1-/-embryos were prepped at E14.5. Ventral midbrain was isolated and snap-frozen in liquid nitrogen. Tissue extraction was performed with RIPA buffer (Sigma, R0278) supplemented with 1x Halt protease \& phosphatase inhibitor (Thermofisher Scientific, 78442) on ice according to the manufacturer's instructions. Protein concentrations were determined by BCA assay (Thermofisher Scientific) using a BSA calibration curve. Protein supernatant was mixed with $4 x$ LDS buffer and loaded on a 4-12\% Bis Tris gel (NUPAGE, NP0335BOX). Protein was blotted on a PVDF membrane, blocked for $1 \mathrm{~h}$ at RT and incubated with primary antibody overnight. After washing with TBST, membrane was incubated with a corresponding horse radish peroxidase (HRP) coupled secondary antibody. Membrane was washed with TBST and visualization of immunoreactive proteins was conducted with a chemiluminescent HRP substrate solution (Super signal femto, Thermofisher Scientific/ Western HRP substrate, Merck Millipore) using a chemiluminescent imager (Chemidoc, Bio-Rad). Bound proteins were removed using $1 \times$ Western blot stripping buffer ( $2 \%$ SDS, 60,02 mM Tris ( $\mathrm{pH}$ 6.8), $100 \mathrm{mM}$ ß-mercaptoethanol) and immunodetection was repeated. For quantification, densitometric analysis was performed, normalization was carried out with total protein (Amido Black, Sigma Aldrich) using the software Image Lab (Bio-Rad).

\section{In situ hybridization}

Sections were post-fixed in 4\% PFA for 10 min, rinsed in PBS and acetylated in $0.1 \mathrm{M}$ TEA (triethanolamine) $\mathrm{HCl}$ with $125 \mu \mathrm{L}$ acetic anhydride for $5 \mathrm{~min}$ with stirring. Sections were washed in PBS and briefly dehydrated in 70\%, 95\% and 100\% ethanol (EtOH). $1 \mu \mathrm{g}$ of RNA probe was added to 1 $\mathrm{mL}$ hybridization buffer and incubated for $2 \mathrm{~min}$ at $80^{\circ} \mathrm{C}$. Sections were air-dried and transferred to a humidified hybridization cassette. A 1:1 mixture of formamide and $\mathrm{H} 2 \mathrm{O}$ was used as humidifying solution. $300 \mu \mathrm{L}$ hybridization solution containing RNA probe was added to each slide, slides were covered with RNase-free coverslips and incubated at $55^{\circ} \mathrm{C}$ overnight. On the following day, coverslips were removed in prewarmed $5 X$ SSC. To reduce unspecific hybridization, sections were incubated in a 1:1 solution of formamide and 2 XSC (high stringency wash solution) for $30 \mathrm{~min}$ at $65^{\circ} \mathrm{C}$. Sections were then washed with RNAse buffer, containing $0.1 \%$ RNase A at $37^{\circ} \mathrm{C}$ for $10 \mathrm{~min}$ to remove non-hybridized RNA. Sections were washed twice with high stringency solution for 20 min at $65^{\circ} \mathrm{C}$, once with $2 \times \mathrm{SSC}$ and once with $0.1 \times \mathrm{SSC}$ for $15 \mathrm{~min}$ at $37^{\circ} \mathrm{C}$. Sections were placed in a humidified chamber and incubated with $10 \%$ normal goat serum in $0.1 \%$ PBS-Tween (blocking solution) for 1 hour at RT. Sections were incubated with anti-DIG-AP Fab fragments (diluted 1:5000 in $1 \%$ goat serum in $0.1 \%$ PBS-Tween) for $3 \mathrm{~h}$ at RT, or overnight at $4^{\circ} \mathrm{C}$. Sections were washed several times $0.1 \%$ PBS-Tween, followed by two washes in NTMT buffer (containing $1 \mathrm{mg} / \mathrm{mL}$ levamisole to reduce background of endogenous alkaline phosphatase activity) for $10 \mathrm{~min}$ at RT. Sections were incubated in BM purple, a substrate for alkaline phosphatase (with $0.5 \mathrm{mg} / \mathrm{mL}$ levamisole) at RT until signal was observed. The chromogenic reaction was stopped by a 10 min incubation in TE buffer at RT. Sections were then washed in PBS, and immunostained for TH.

\section{Image acquisition of fixed cryosections}


Table 2. Antibodies used for immunohistochemistry and western blots

\begin{tabular}{|c|c|c|}
\hline \multicolumn{3}{|c|}{$\begin{array}{l}\text { Immunohistochemistry: Primary antibodies } \\
\text { (made in donkey) }\end{array}$} \\
\hline Antibody & Manufacturer & $\begin{array}{l}\text { Concentra- } \\
\text { tion }\end{array}$ \\
\hline rabbit anti-TH & AB152 Merck & $1: 500$ \\
\hline mouse anti-TH & MAB318 Merck & $1: 500$ \\
\hline rabbit anti-Girk2 & APC006 Alamone Labs & $1: 400$ \\
\hline rabbit anti-Calbindin & C9848 Swant & 1: 5000 \\
\hline rabbit anti-GFP & A11122 Life Technologies & 1: 400 \\
\hline rat anti-GFP & 04404-84 Nalacai & 1: 1500 \\
\hline goat anti-Reelin & AF3820 R\&D systems & 1: 50 \\
\hline rabbit anti-Dab1 & $\begin{array}{l}\text { kindly provided by Dr. Brian } \\
\text { Howell }\end{array}$ & $\begin{array}{l}\text { 1: } 5000 \\
\text { (TSA kit) }\end{array}$ \\
\hline goat anti-Otx2 & GT15095 Neuromics & 1: 5000 \\
\hline rabbit anti-Sox6 & ab30455 Abcam & 1: 500 \\
\hline rabbit anti-N-Cadherin & ab18203 Abcam & $1: 250$ \\
\hline \multicolumn{3}{|l|}{ Immunohistochemistry: } \\
\hline \multicolumn{3}{|c|}{ Secondary antibodies (made in donkey) } \\
\hline anti-rabbit Alexa 488 & A21206 Invitrogen & 1: 500 \\
\hline anti-rabbit Alexa 350 & A10039 Invitrogen & 1: 500 \\
\hline anti-mouse Alexa 488 & A21202 Invitrogen & 1: 500 \\
\hline anti-rat Alexa 488 & A21208 Molecular probes & 1: 500 \\
\hline anti-goat Alexa 488 & $\begin{array}{l}\text { 705-545-147 Jackson } \\
\text { ImmunoResearch }\end{array}$ & 1: 500 \\
\hline anti-rabbit Cy3 & $\begin{array}{l}\text { 711-165-152 Jackson } \\
\text { ImmunoResearch }\end{array}$ & 1: 200 \\
\hline anti-mouse Cy3 & $\begin{array}{l}\text { 715-165-150 Jackson } \\
\text { ImmunoResearch }\end{array}$ & 1: 200 \\
\hline anti-goat Cy3 & $\begin{array}{l}\text { 705-165-147 Jackson } \\
\text { ImmunoResearch }\end{array}$ & 1: 200 \\
\hline biotinylated donkey anti-rabbit & $\begin{array}{l}\text { 711-065-152 Jackson } \\
\text { ImmunoResearch }\end{array}$ & 1: 200 \\
\hline \multicolumn{3}{|l|}{ Western blot } \\
\hline rabbit anti-cofilin & $\begin{array}{l}\text { Kindly provided by Dr. Prof. } \\
\text { Walter Wittke }\end{array}$ & $1: 5000$ \\
\hline rabbit anti-p-Cofilin (ser3) (77G2) & \#3313 Cell signaling & $1: 1000$ \\
\hline rabbit anti-p-Limk1 (Thr508) & \#3841 Cell signaling & $1: 500$ \\
\hline \multicolumn{3}{|l|}{ /Limk2 (thr505) } \\
\hline Rabbit anti-Limk1 & \#3842 Cell signaling & $1: 1000$ \\
\hline Rabbit anti-N-cadherin & Abcam ab18203 & $1: 500$ \\
\hline $\mathrm{Rb}-\mathrm{HRP}$ & \#7074 Cell signaling & $1: 1000$ \\
\hline
\end{tabular}

Embryonic and adult sections were imaged at an inverted Zeiss AxioObserver Z1 microscope equipped with an ApoTome. Fluorescence images were acquired with Zeiss AxioCam MRm $1388 \mathrm{x}$ 1040 pixels (Carl Zeiss). At 10X (EC PInN 10x/0.3, Carl Zeiss) and 20X (EC PInN 20x/0.5, Carl Zeiss) magnifications, tile images were acquired with conventional epifluorescence. ApoTome function was used to acquire tile images and z-stacks at 40X (PIn Apo 40x/1.3 Oil, Carl Zeiss) and 63X (PIn Apo 63x/1.4 Oil, Carl Zeiss) magnifications. In situ hybridized sections were imaged with transillumination (AxioCam MRc, $1300 \times 1030$ pixels, Carl Zeiss) at the AxioObserver Z1 setup. Images 
were stitched with Zen blue software (Zeiss, 2012). Sections stained with Alexa 649 secondary antibody, and 63X confocal images were imaged at a Leica SP8 confocal microscope and stitched with Leica PC suite (Leica, 2014)

\section{Organotypic slice culture and time lapse imaging}

Organotypic slice cultures were generated as previously described Bodea and Blaess (2012). Slices were placed on Millicell membrane inserts (Merck) and incubated for $6-12 \mathrm{~h}$ at $37^{\circ} \mathrm{C}, 5 \% \mathrm{CO}_{2}$, before imaging. Slices were briefly examined at a Zeiss Axioobserver microscope with conventional epifluorescence. Healthy slices, with well-defined, strongly fluorescent cells, were chosen for twophoton excitation imaging. Slices on their membrane inserts were transferred to $\mu$-Dish imaging dishes (Ibidi) containing $750 \mu \mathrm{L}$ of prewarmed, fresh culture medium ( $5 \mathrm{~mL}$ Hank's balanced salt solution, $9 \mathrm{~mL}$ DMEM high glucose (Sigma Aldrich), $5 \mathrm{~mL}$ horse serum, $200 \mu \mathrm{L}$ Penicillin/Streptomycin for $20 \mathrm{~mL}$ of culture medium). Slices were imaged at 32X magnification (C-Achroplan 32x/0.85, Carl Zeiss) with an inverted, two-photon Zeiss LSM 710 NLO microscope, equipped with temperature and $\mathrm{CO}_{2}$ control (Pecon). The microscope setup and the 32X water immersion objective were preheated for 8 hours before time-lapse experiments. Images were acquired using 920 $\mathrm{nm}$ for excitation with a laser power of $5-10 \%$ (Laser: Chameleon Ultrall, Coherent). A total of 3 control (Shh CreER/+, Rosa26 $6^{\text {lox-stop-loxY FP/+ }}, \mathrm{Dab}^{+/+}$or Shh $\mathrm{CreER} /+^{+}$, Rosa26 $6^{\text {lox-stop-loxY FP/+}}, \mathrm{Dab} 1^{\text {del } /+}$ )

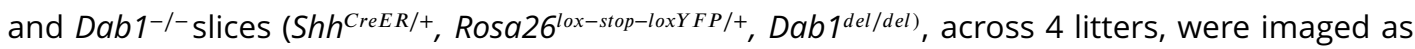
described. Of the 6 slices analyzed, 3 control and 2 Dab1-/- were imaged for 4.3 hours while one Dab1/-I slice was imaged for 2.6 hours. All imaged slices were post-stained with TH to confirm that the region imaged was within the dopaminergic domain Bodea et al. (2014). Organotypic slice cultures were fixed in 4\% PFA for $1 \mathrm{~h}$ at RT, then rinsed in PBS and $0.3 \%$ PBT for $10 \mathrm{~min}$. Slices were incubated in blocking solution (10\% NDS in $0.3 \%$ PBT) at RT for $2 \mathrm{~h}$, or overnight at $4{ }^{\circ} \mathrm{C}$. After blocking, slices were incubated with primary antibody solution (3\% NDS in $0.3 \%$ PBT) for $24-48 \mathrm{~h}$ at $4{ }^{\circ} \mathrm{C}$. The following primary antibodies and dilutions were used: rabbit anti-TH (1:500), rat anti-GFP (1:1000). Slices were washed in $0.3 \%$ PBT and then incubated in secondary antibody solution (3\% NDS in $0.3 \%$ PBT), at RT for $4 \mathrm{~h}$, or overnight at $4^{\circ} \mathrm{C}$. Secondary antibodies donkey anti-rabbit Cy3 (1:200) and donkey anti-rat Alexa 488 (1:500) were used. All steps were carried out in a 6-well plate.

\section{Immunostaining and clearing of whole mount embryonic brains}

Brains from E13.5 embryos were fixed in 4\% PFA for $4 \mathrm{~h}$ at room temperature, or overnight at $4^{\circ} \mathrm{C}$. Brains were washed with PBS, $0.3 \%$ PBT, and incubated with blocking solution (10\%NDS in $0.3 \%$ PBT) overnight at $4^{\circ} \mathrm{C}$. The brains were incubated with primary antibodies: rabbit anti-TH (1:500) and rat anti-GFP (1:1000) at $4^{\circ} \mathrm{C}$ for 2 days. Next, the primary antibody solution was removed and the brains were washed three times with $0.3 \%$ PBT at RT for $15 \mathrm{~min}$. The tissue was incubated with secondary antibodies: donkey anti-rat IgG-DyLight 647 (1:100) and donkey anti-rabbit Cy3 (1:200) at RT for 1 day. Subsequently, the tissue was washed three times with $0.3 \%$ PBT and three times with PBS for $20 \mathrm{~min}$. All washing steps and antibody solutions preparation were performed using $0.3 \%$ PBT. All steps were carried out in 24-well plates.

Tissue clearing was carried out as described previously Schwarz et al. (2015). The procedure was modified for embryonic tissue as described here: After immunostaining, brains were incubated in increasing concentrations $(30 \%, 50 \%, 70 \%)$ of tert-butanol $(\mathrm{pH} 9.5)$ for $4 \mathrm{~h}$ at RT followed by $96 \%$ and $100 \%$ tert-butanol $\left(\mathrm{pH} \mathrm{9.5)}\right.$ for $4 \mathrm{~h}$ at $33^{\circ} \mathrm{C}$. Brains were then incubated overnight in a triethylamine $\mathrm{pH}$-adjusted 1:1 mixture of benzyl alcohol/benzyl benzoate $(\mathrm{BABB}, \mathrm{pH} 9.5)$ at $33^{\circ} \mathrm{C}$. Brains were stored in clearing solution at $4^{\circ} \mathrm{C}$ and imaged within 1 week of clearing. Whole mount brains were imaged in clearing solution with a 20X BABB dipping objective (Olympus) at a Leica SP8 upright microscope. Multi-channel image acquisition of the whole brain (4-6 tiles, $150-200$ z-steps, step-size $=1.5 \mu \mathrm{m}$ ) took $30-70 \mathrm{~h}$, and resulted in multichannel datasets of large sizes (20 $80 \mathrm{~GB}$ ). Voxel size of thus acquired images was $0.432 \mu \mathrm{m} \times 0.432 \mu \mathrm{m} \times 1.5 \mu \mathrm{m}$. Individual tiles at each z-step were stitched together using the Leica SP8 PC suite (Leica, 2014).

\section{Analysis of cell distribution in fixed slices}

Mediolateral distribution of mDA neurons was analyzed for $n>3$ animals at each time point of 
analysis (E15.5, E18.5 and P21-30) by constructing normalized bins spanning the entire TH-positive domain. Since we observed that in both, Dab1 CKO and Dab1 -/- mice, a few TH-positive cells of the lateral most SN lateralis were consistently present (yellow arrowheads (Figure $1 \mathrm{D}, \mathrm{E}, \mathrm{H}, \mathrm{I}$ ), we defined the mediolateral bins by quadrisecting a line extending from the midline to the lateral most TH positive cells (Figure 1B). The fraction of the total number of TH positive cells in each mediolateral bin was evaluated for control, Dab1 CKO and Dab1-/- brains.

\section{Speed and trajectories of migrating $\mathrm{mDA}$ neurons}

To prevent any bias in selection of cells for tracking, and to track a large number of neurons in 3D in our two-photon time lapse datasets, we used the semi-automatic plugin TrackMate in Fiji Tinevez et al. (2017). Before soma detection, a $3 X 3$ median filter was applied by the TrackMate plugin, to reduce salt and pepper background noise. Soma detection was carried out using the Laplacian of Gaussian (LoG) detector in TrackMate. The soma detected by the TrackMate plugin were automatically linked across time, in 3D, by using the linear assignment problem (LAP) tracker in TrackMate Tinevez et al. (2017); Jaqaman et al. (2008). After automatic tracking, the track scheme view in TrackMate was used to check the accuracy of each track by eye. Spurious tracks were deleted and missed detections were added using the manual tracking mode in TrackMate. Excel files from the TrackMate plugin were imported into MatLab Wu et al. (2015). 3D soma velocity was obtained at every time point (in units of $\mu \mathrm{m} / \mathrm{hr}$ ) of the analysis (starting $\mathrm{t}=10 \mathrm{~min}$ ) as the change in soma position vector between the previous frame and the current frame, divided by the time duration (0.167 hr) between frames (code modified from Wu et al. (2015)). This data was used to generate probability histograms for average soma speed, maximum soma speed, time spent at rest (defined as soma speed $<10 \mu \mathrm{m} / \mathrm{hr}$ ), time spent in slow migration (soma speed between 10 and $30 \mu \mathrm{m} / \mathrm{hr}$ ), time spent in medium-fast migration (30-60 $\mu \mathrm{m} / \mathrm{hr}$ ) and time spent in fast migration (soma speed > $60 \mu \mathrm{m} / \mathrm{hr}$ ). Categories for rest, slow, medium-fast and fast speeds were defined for the purpose of easy visualization of data, and were based on $25 \%$ percentile $(10 \mu \mathrm{m} / \mathrm{hr})$ and $75 \%$ percentile speeds (30 $\mu \mathrm{m} / \mathrm{hr}$ ) of $D a b 1^{-/-}$population.

Cell trajectory angles were measured in $2 \mathrm{D}$ as the angle between midline (positive $\mathrm{y}$-axis in the image) and the line joining the first and final soma positions. Cells that moved with maximum speeds of less than $10 \mu \mathrm{m} / \mathrm{hr}$ were excluded from the trajectory analysis as they were categorized as being at rest. Statistics on trajectory angles were performed with CircStat: a MatLab toolbox Berens (2009).

Only cells for which the soma were detected at all time points of imaging were included in the analysis. Using this approach, we tracked 806 cells in slices from control mice (Shh ${ }^{\text {CreER/+ }}$,

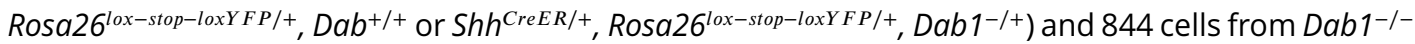

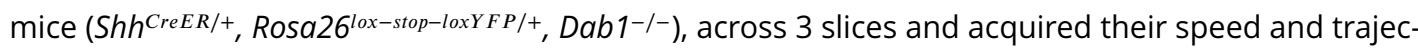
tory profiles. Each cell (and track) had a unique ID assigned by the TrackMate plugin. These cell IDs were used to identify and locate individual cells in the slice for further analysis.

\section{Morphology analysis of migrating mDA neurons}

We restricted our morphological analysis to $n=150$ control (70 fast, 40 medium-fast and 40 slow cells), and 129 Dab1-/-(49 fast, 40 medium-fast and 40 slow) cells. We observed that Dab1-/- cells continuously extended neurites in slices and this made it difficult to unambiguously assign processes to individual cells as imaging progressed. Hence, we examined the morphology of each cell, in 3D, for the first 18 frames of imaging. Cell soma was defined as the spot detected/assigned to the cells in the TrackMate plugin. Analysis was done manually, by rendering individual neurons in 4D (3D projection over all time frames) in Image, and recording the number of primary processes (arising from the soma) and secondary processes at each time point. A cell was defined as bipolar when fewer than two processes were observed arising directly from the soma. The appearance/ disappearance of any branch was regarded as a branch transition. At each time point, the morphology of the cell, and the number of branch transitions, was manually annotated to the spot position data of the cell in excel sheets exported from TrackMate. In addition, 20 control and Dab1-/- cells were randomly chosen for tracing in 3D. These neurons were traced manually in simple neurite tracer (SNT) plugin 
of Fiji Longair et al. (2011). Tracings were carried out, at each time point individually, for the first 18 frames of imaging. Fills of traced neurons were generated semi-automatically in the SNT plugin. Fill thickness was decided by eye but was maintained across all time points for a cell. Maximum intensity projections were also generated for the 3D segmentation fills. SNT traces were also used to measure length of the leading process in 3D.

\section{Statistical analysis}

Statistical significance of mediolateral distributions of $\mathrm{TH}^{+}$mDA neurons in control, Dab1 CKO and $\mathrm{Dab1} 1^{-/-}$adult and embryonic brains were assessed by two-way ANOVA with Tukey's correction for multiple comparisons ( $n=6$ animals/ genotype, at P30 and $n=4$ animals/ genotype at E18.5). At $\mathrm{E} 15.5$, mediolateral distribution of $\mathrm{TH}^{+} \mathrm{mDA}$ neurons and $\mathrm{P} 30 \mathrm{TH}^{+} \mathrm{GIRK} 2^{+}$mediolateral distributions in control and Dab1 CKO brains were assessed for statistical significance by Student's t-test. All non-parametric distributions were analyzed with Mann-Whitney's non-parametric rank test, KruskalWallis test or Kalmogrov-Smirnov test (mentioned in figure legends) in Prism 7/ MatLab. Circular variables were analyzed with the CircStat toolbox for MatLab. Angle distribution in populations were compared using Kuiper's test for circular variables Berens (2009) .

\section{Acknowledgements}

This work was supported by the Maria von Linden-Program and BONFOR (both University of Bonn, to S.B.), the German Research Foundation (BL 767/2-1, BL 767/3-1 to S.B.), a German Academic Exchange Service doctoral fellowship (to A.R.V) and a European Union grant (FP7-HEALTH-F4-2013602278-Neurostemcellrepair to O.B.). We thank Brian Howell for providing the Dab1 antibody; Walter Witke for providing the Cofilin1 antibody, Nils-Görran Larsson for the DAT ${ }^{\text {Cre }}$ mouse line; Ulrich Müller for providing the Dab1 $1^{\text {del }}$ and $D a b 1^{\text {flox }}$ mouse lines; Donato Di Monte and Michael Helwig for assistance with two-photon imaging; Jonas Doerr, Martin Schwarz and Anke Leinhaas for initial support with clearing and imaging of whole-mount brains; Petra Mocellin for initial support with image analysis; Killian Berendes for technical support; Gabriela Bodea, David Greenberg and Marianna Tolve for critical reading of the manuscript. The authors declare no competing financial or non-financial interests. 

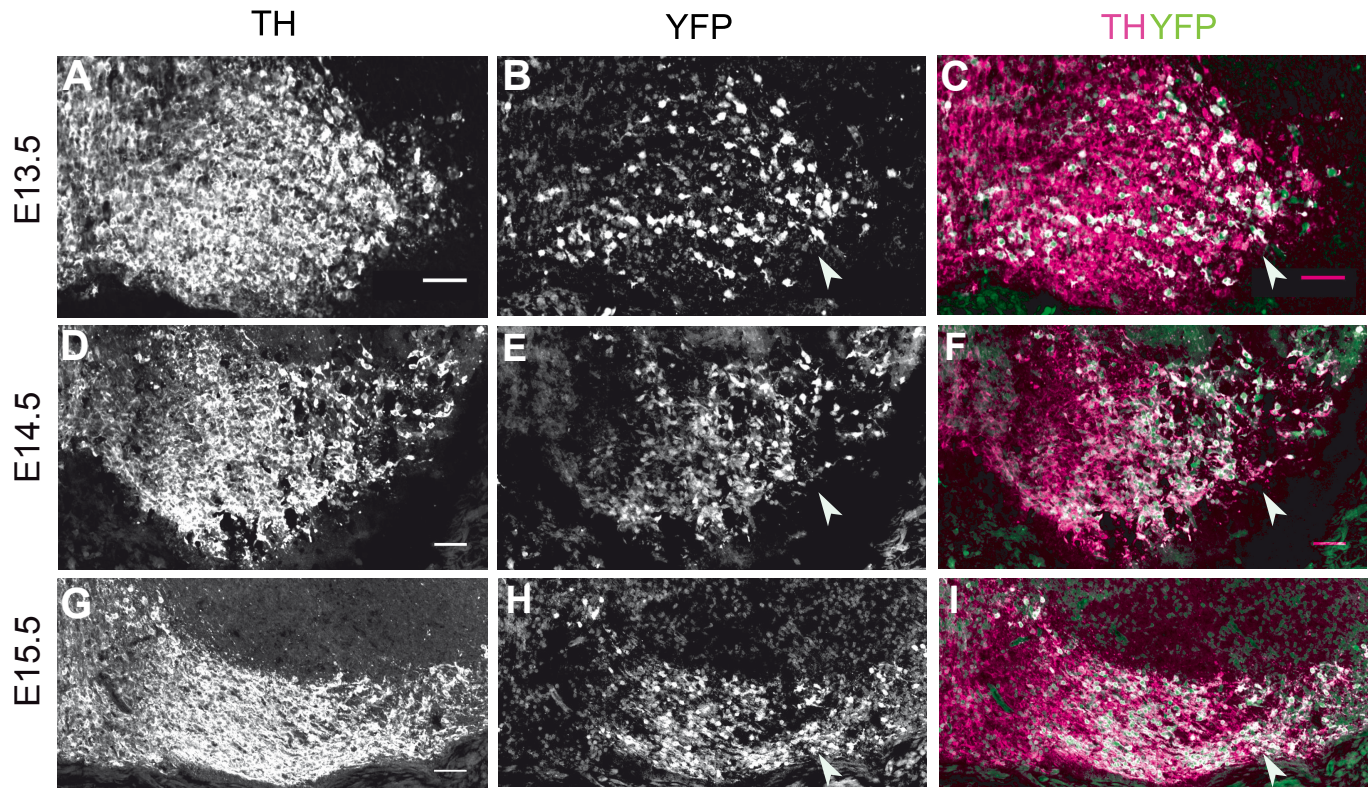

Figure 1- figure supplement 1: Dat ${ }^{\text {Cre }}$ mediated recombination pattern. (A-C) Analysis of Cre-mediated recombination in DAT Cre/+, Rosa26 EYFP/+ mice. Immunostaining for TH and YFP at E13.5 shows Cre-mediated recombination in the lateral mDA regions (white arrowhead). (D-F) Immunostaining for $\mathrm{TH}$ and YFP at E14.5 shows almost complete recombination in mDA neurons of the SN (white arrowhead). (G-I) By E15.5, $\mathrm{TH}^{+}$mDA neurons of the SN and lateral VTA are YFP ${ }^{+}$

(white arrowhead). Scale bar: $50 \mu \mathrm{m}$. 
TH
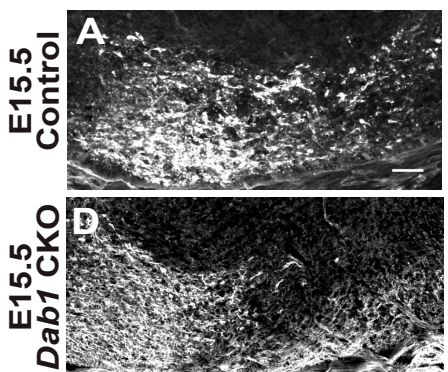

Figure 1- Figure supplement 2. Specific loss of DAB1 protein in mDA neurons

of Dab1 CKO brains. (A-C) Analysis of DAB1 protein expression in control mice at E15.5: Immunostaining for TH and DAB1 at E15.5 shows DAB1 expression in $\mathrm{TH}^{+}$ neurons of the SN and lateral VTA (white arrowheads) and in non-dopaminergic regions lateral to $\mathrm{mDA}$ neurons (yellow arrowhead). (D-F) Analysis of DAB1 expression in Dab1 CKO mice at E15.5 shows absence of DAB1 expression in $\mathrm{TH}^{+}$ $\mathrm{mDA}$ neurons (white arrowheads), while DAB1 expression persists in lateral nondopaminergic regions (yellow arrowhead). Scale bar: $50 \mu \mathrm{m}$. 
TH, E15.5 Control

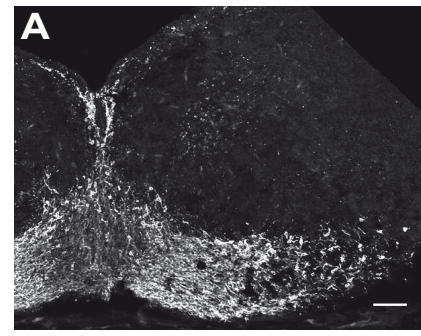

TH, E15.5 Dab1 CKO

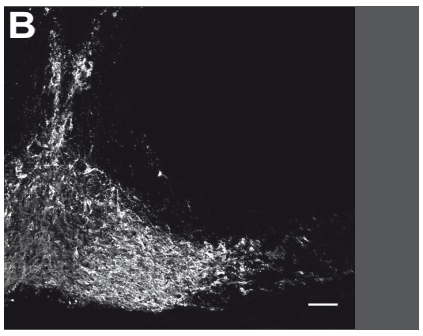

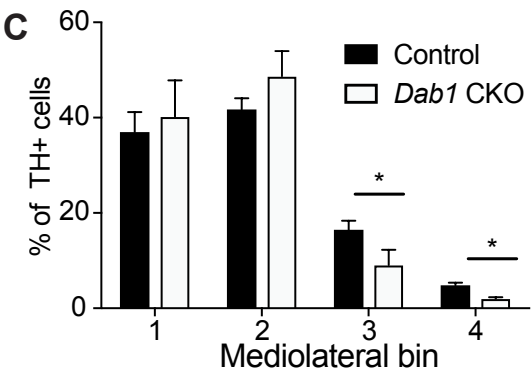

Figure 1- Figure supplement 3. Mediolateral distribution of mDA neurons at E15.5 in absence of Reelin signaling. Analysis of mediolateral distribution of $\mathrm{TH}^{+}$cells in control $(A)$ and Dab1 CKO mice (B) at E15.5 shows a medial accumulation of cells in Dab1 CKO mice (C). Data are represented as mean + s.e.m for $n=3$ brains. ${ }^{*}$ indicates $p<0.05$ by Student's t-test corrected for multiple comparisins (Holm-Sidak method). Scale bars: $50 \mu \mathrm{m}$. 
A
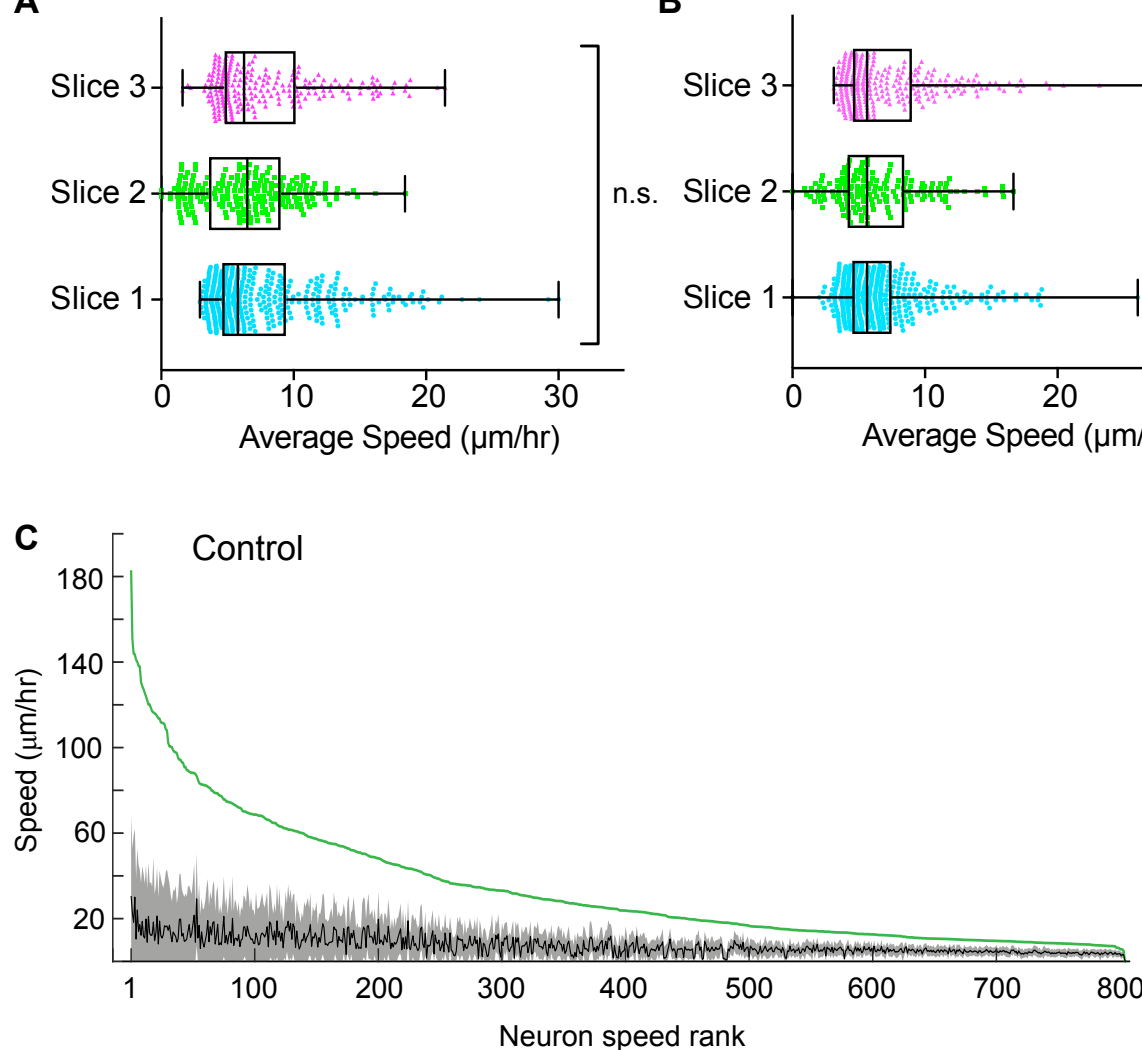

D

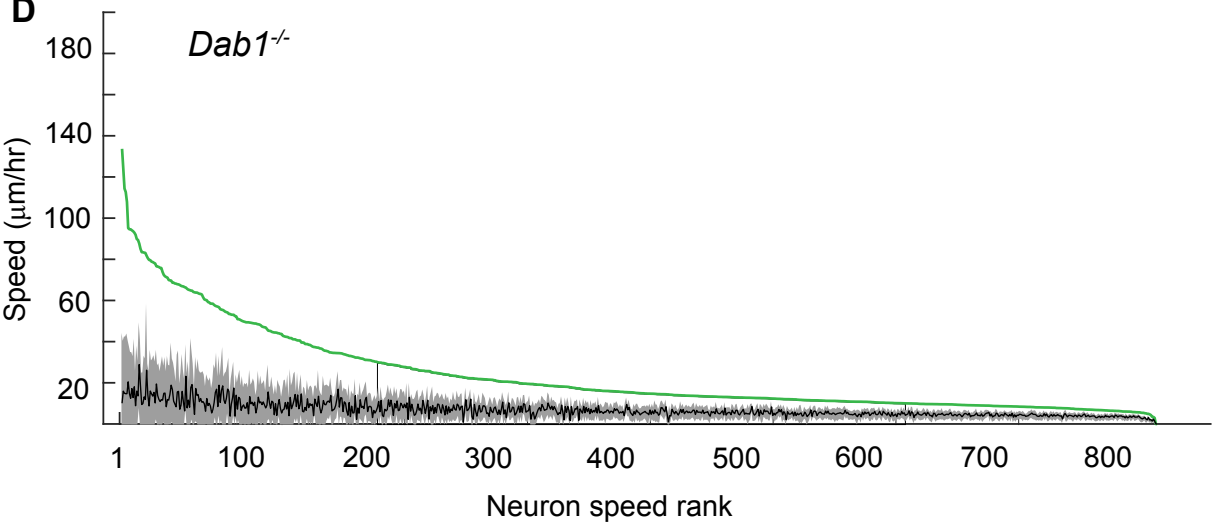

B

Dab1-

n.s.

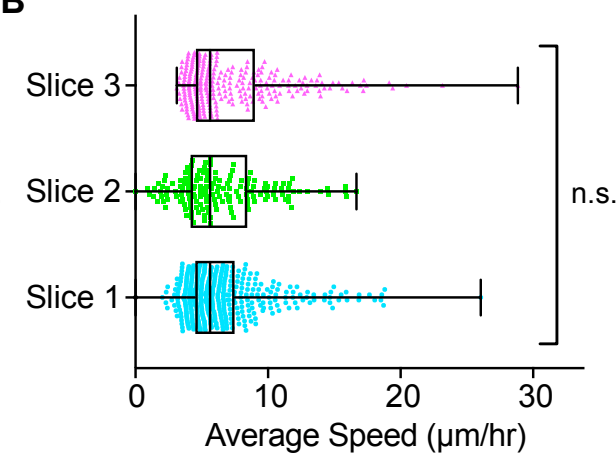

Figure 4- Figure supplement 1. Average speed distributions and speed profiles of SN-mDA neurons. (A) Average speed distributions across 3 control slices are not significantly different $(p=$ 0.1065 , Kruskal-Wallis test for non-parametric distributions). (C) Average speed distributions across 3 Dab1\% slices are not significantly different $(p=0.2279$, Kruskal-Wallis test for non-parametric distributions). (C,D) To demonstrate the extent of max-speed variation in control and Dab1/ mDA neurons from their mean speeds, each neuron in the dataset was ranked by its max-speed (green curve for the population). For each neuron, the mean and standard deviation around the mean were also plotted: grey and black curves represent variation of soma speed (grey) around average speed (black) for all mDA neurons in the dataset. 
753

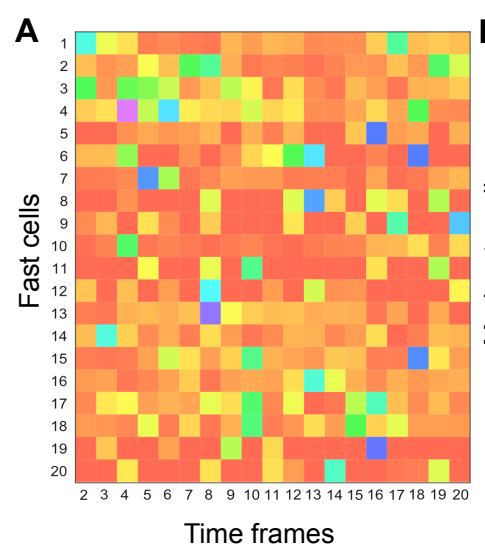

B

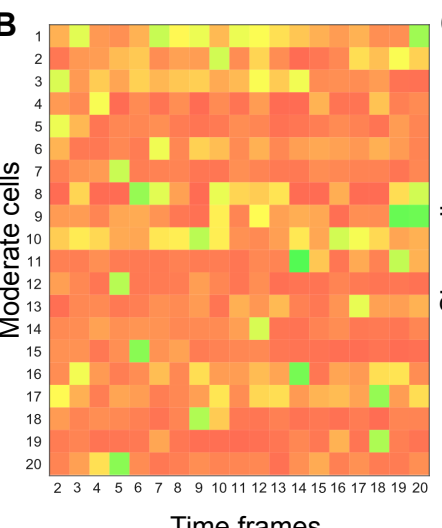

Time frames

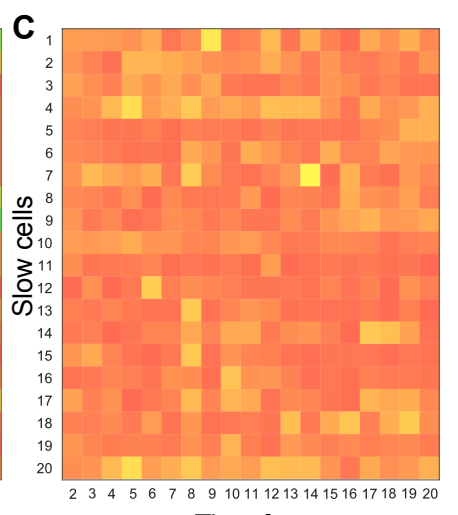

Time frames
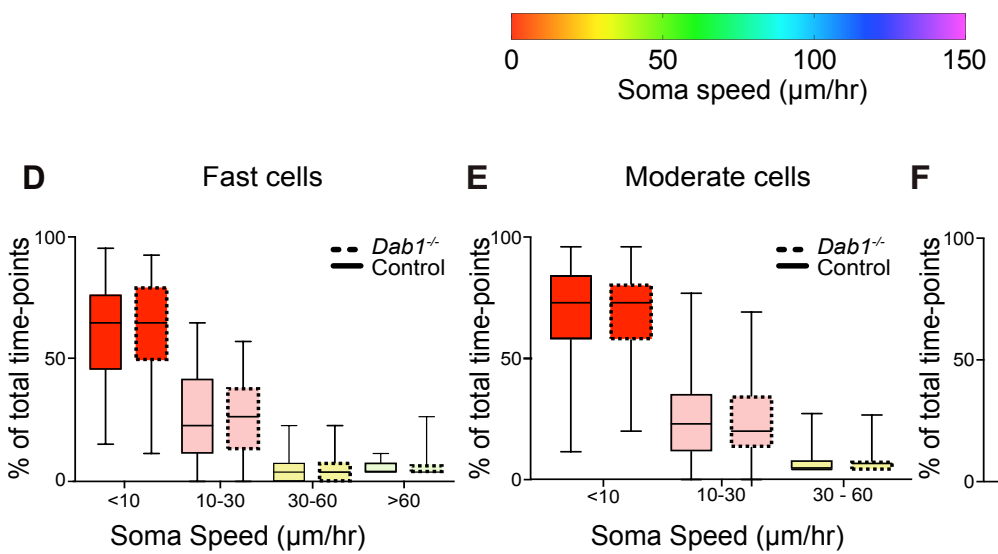

E

Moderate cells

$\mathbf{F}$

Slow cells
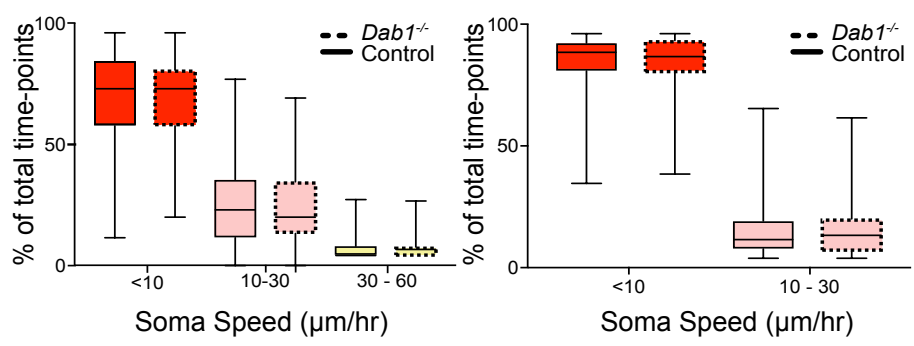

Figure 5 - Figure supplement 1. Variation in instantaneous soma speed of mDA neurons. (A-C) Instantaneous soma speeds of 20 fast, moderate or slow cells in control slices as a heat map across 20 consecutive time frames. Each cell is one row in the heat-map, each column is a time frame. Soma speeds range from $0-150 \mu \mathrm{m} / \mathrm{hr}$. (D-F) Individual fast, moderate and slow cells spend comparable periods of time in fast, moderate, slow and rest phases of migration in Dab1\% slices compared to control slices. 
756 A

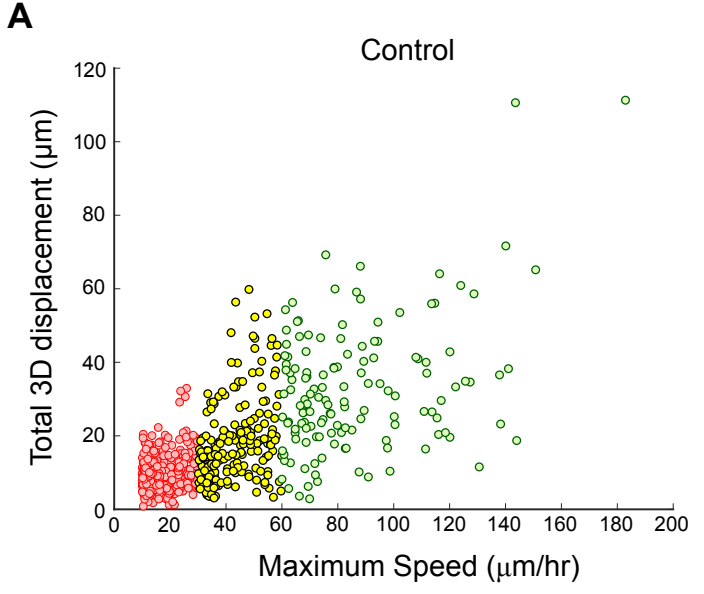

C

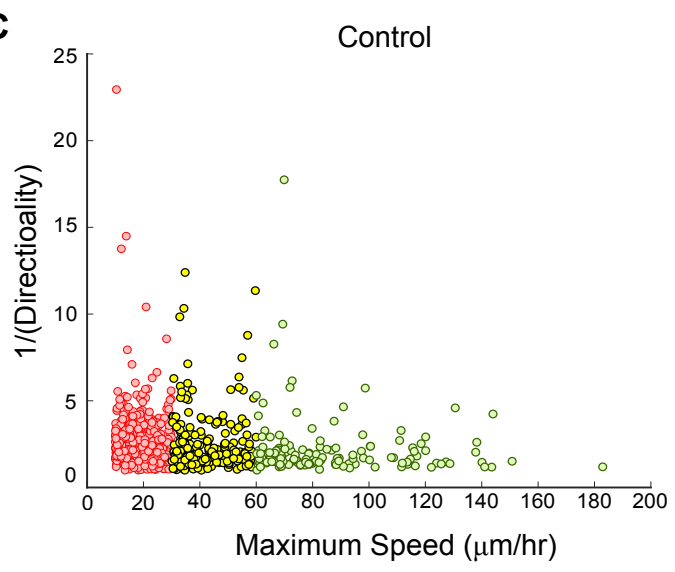

B

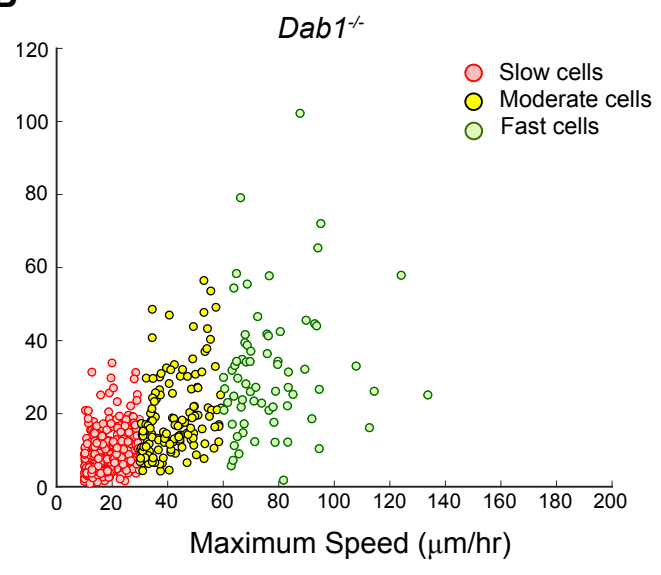

D

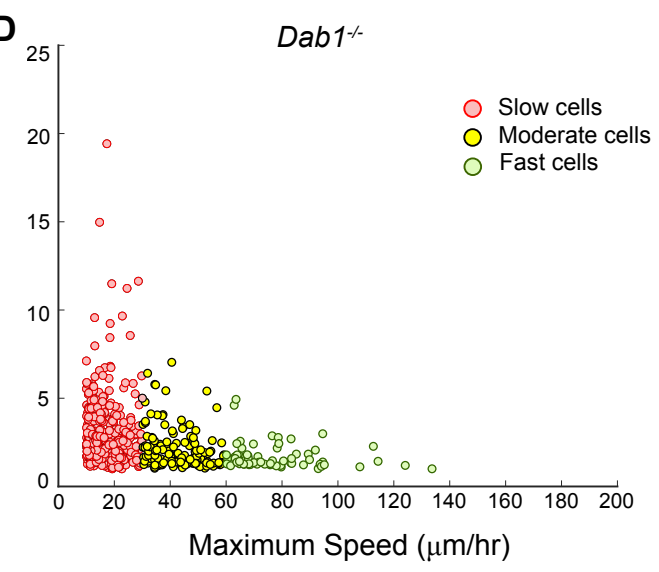

Figure 5- Figure supplement 2. Individual fast, moderate and slow mDA neurons from Dab1r-slices have similar directionality and displacement profiles as mDA neurons in control slices. (A,B) Total displacement (3D) increases with increasing max-speed of mDA neurons in control and Dab1/ slices. (C,D) Directionality of mDA neurons increases with increasing maxspeed in control and $D a b 1^{1 /}$ slices. Note that inverse values of directionality are plotted against the max-speeds of migratory mDA neurons. 
A
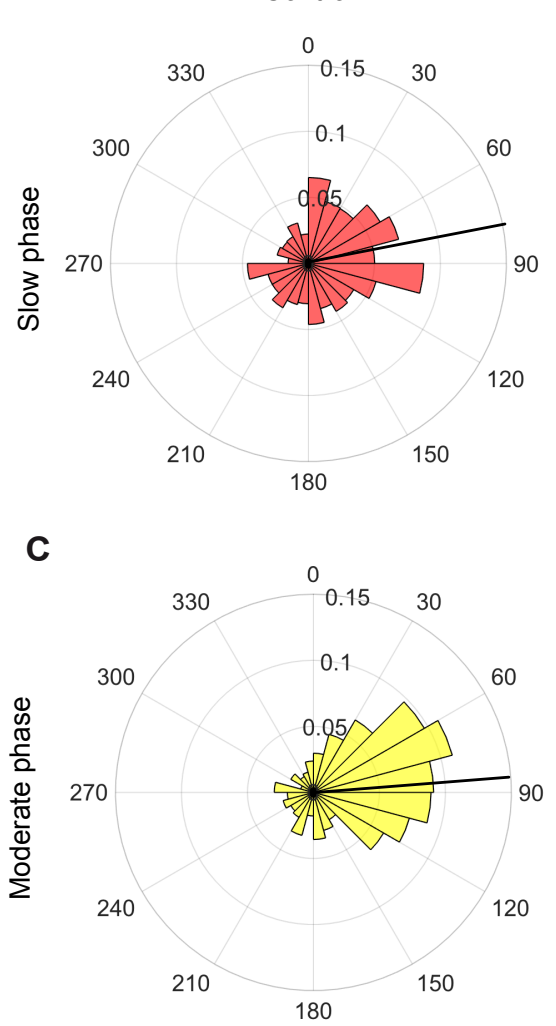

E

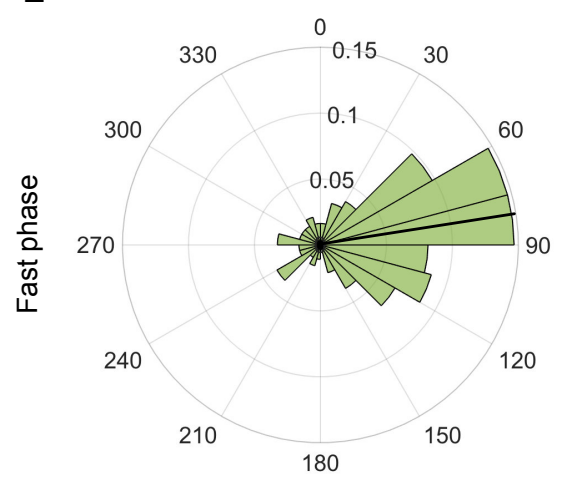

B

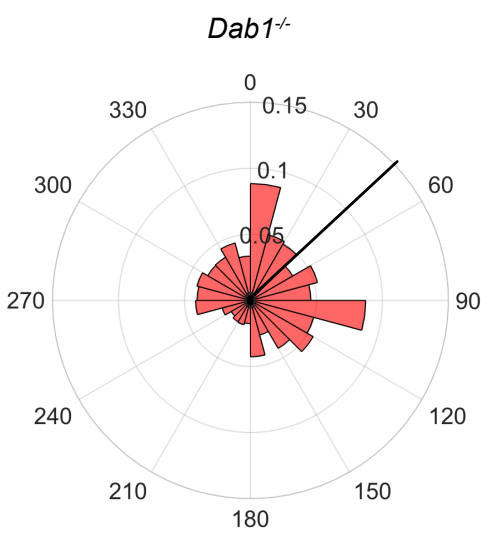

D

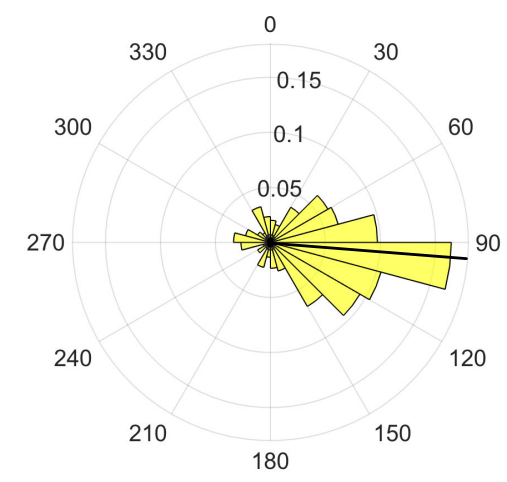

$\mathbf{F}$

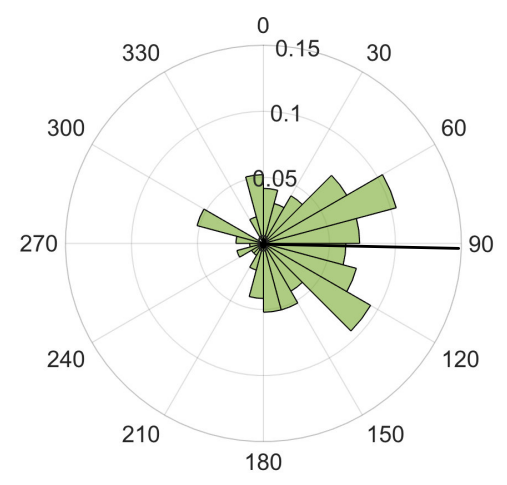

Figure 7 - Figure supplement 1 . Lateral migration occurs predominantly during moderate and fast migratory phases of mDA neurons. (A,B) Polar histogram for angle of slow mDA trajectories to the midline shows that slow migratory movements have the least preference for lateral migratory direction in both control (A) and Dab1- (B) slices $(n=3506$ movements in control, $\mathrm{n}=2622$ movements in Dab1/ mDA neurons). (C-F) Moderate (C,D; $\mathrm{n}$ $=506$ movements in control, $\mathrm{n}=298$ movements in Dab1 1 mDA neurons) and fast movements ( $\mathrm{E}, \mathrm{F} ; \mathrm{n}=184$ movements in control, $\mathrm{n}=96$ movements in $D a b 1^{-}$- $\mathrm{mDA}$ neurons) are laterally directed and comparable in control and Dab1- slices. Red (A,B), black (C,D) and green (E,F) lines represent mean angular direction for slow, moderate and fast populations, respectively. 


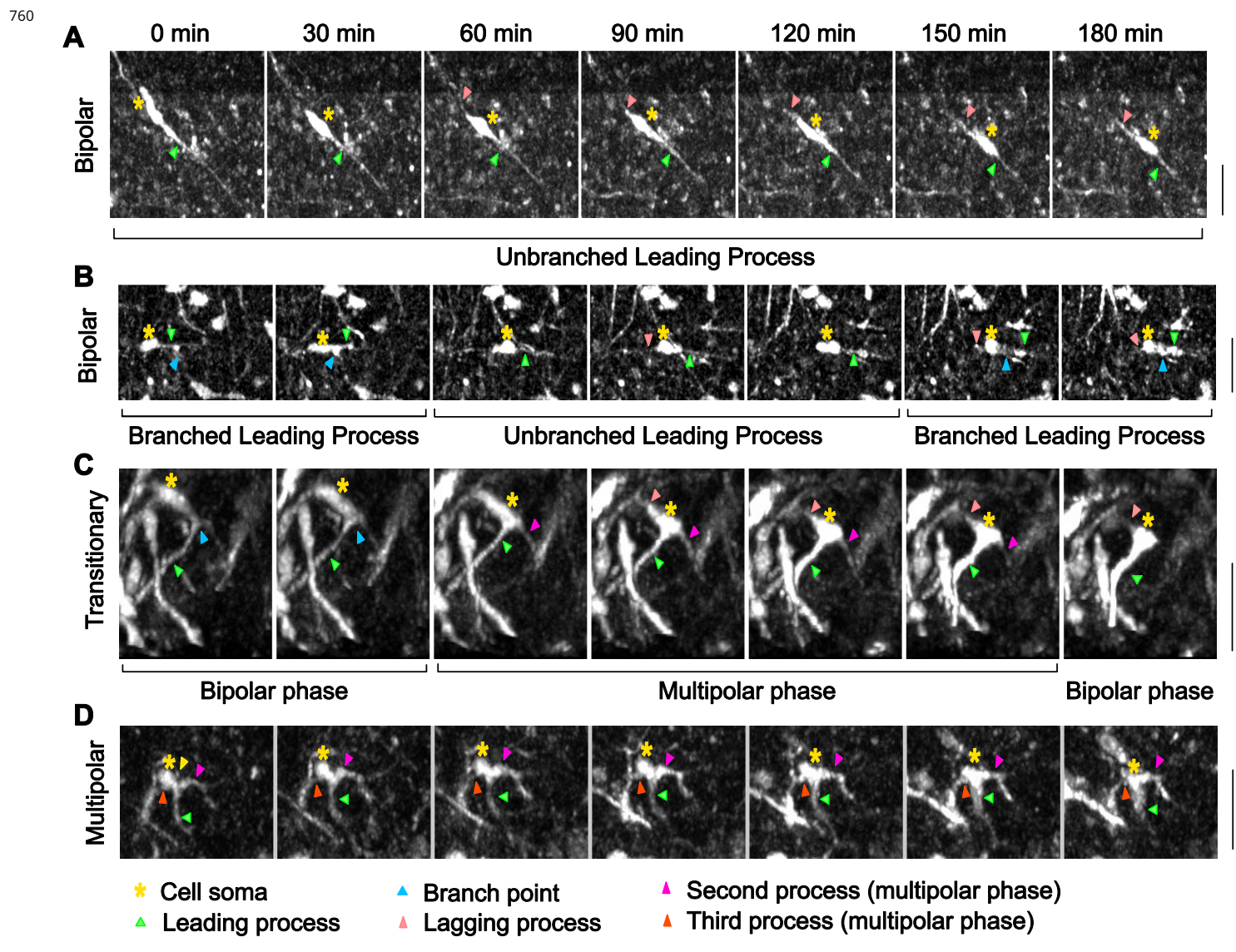

Figure 8 - Figure supplement 1. Morphological characterization of mDA neurons. (A) Control bipolar mDA neuron displaying bipolar-unbranched morphology at all analyzed time-points. (B) Bipolar mDA neuron that switches between a branched and unbranched leading process. (C) Transitionary mDA neuron that switches between bipolar and multipolar phases twice in the duration of analysis. (D) mDA neuron that remains multipolar during the entire duration of analysis. 

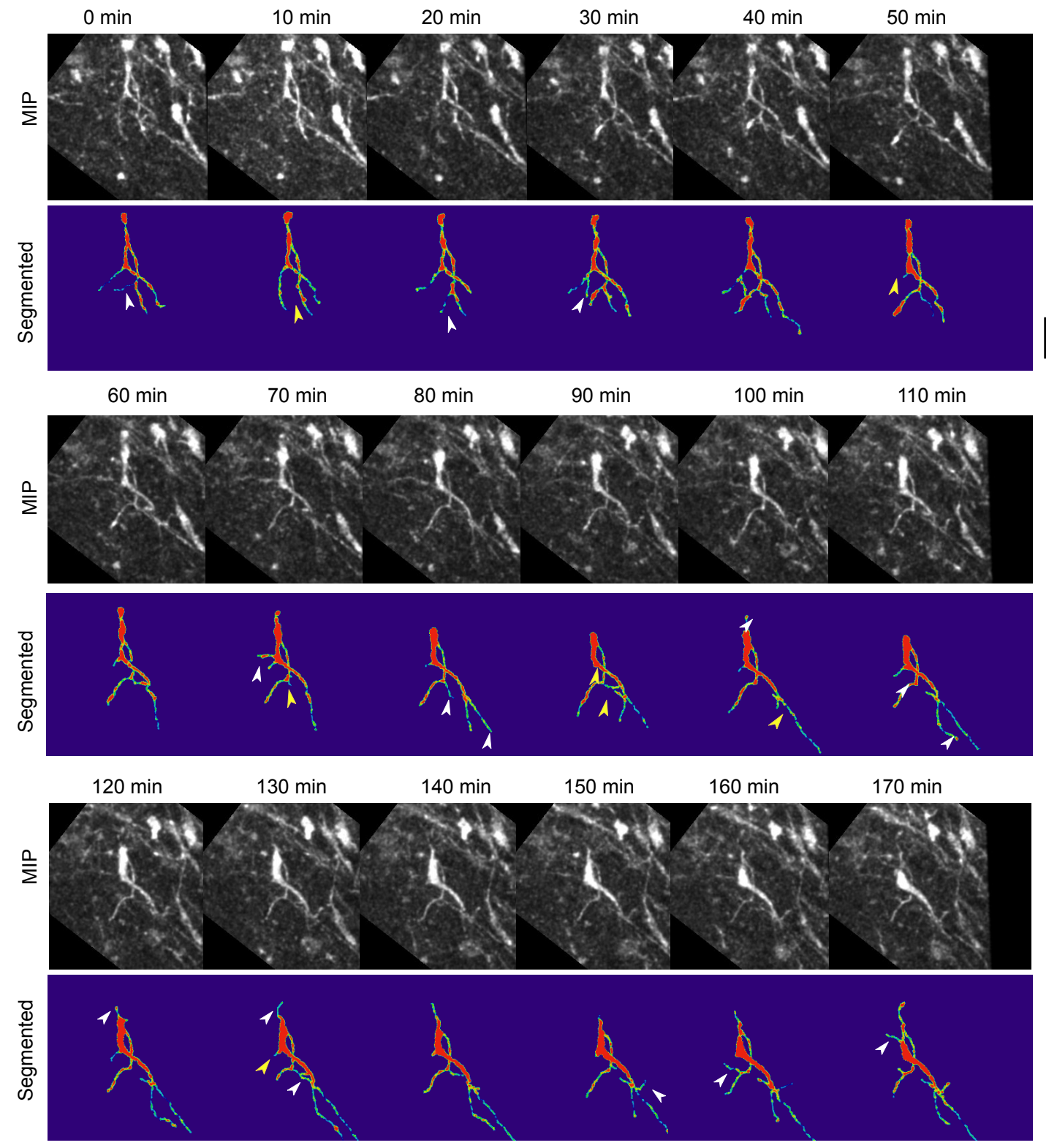

Figure 9 - Figure supplement 1. Absence of Reelin signaling results in the formation of unstable protrusions on the soma and leading process of mDA neurons. Maximum intensity projections of cell shown in Figure 8 for each time point of the analysis. Cell in (A-C) is color-coded for the intensity of YFP in images (Physics LUT in Fiji). Red represents bright structures, green-blue represents weakly labelled structures. White arrowheads indicate appearance of a branch/protrusion, yellow arrowheads indicate disappearance. Scale bar: $25 \mu \mathrm{m}$. 


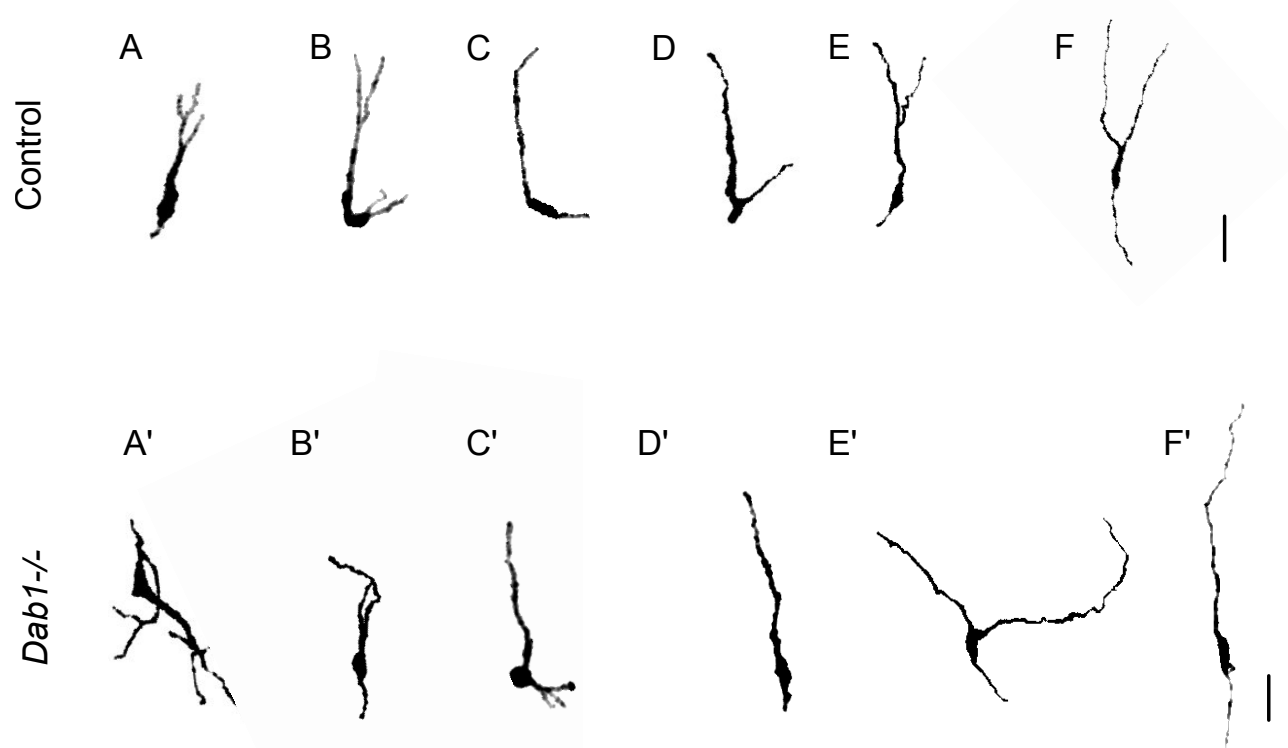

G

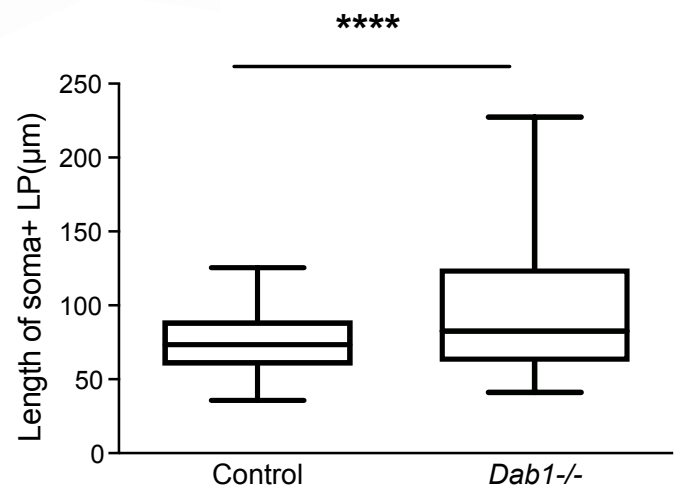

Figure 9 - Figure supplement 2. Greater spread in length of leading process in Dab1-/ mDA neurons. (A-F) 6 of 20 manually segmented control mDA neurons. (A'-F') 6 of 20 manually segmented Dab1/- mDA neurons. (G) Length of cell soma and leading process shows a wider spread in the absence of Reelin signalling. ${ }^{* \star *} p<0.001$ as assessed by Mann-Whitney's test. 
A

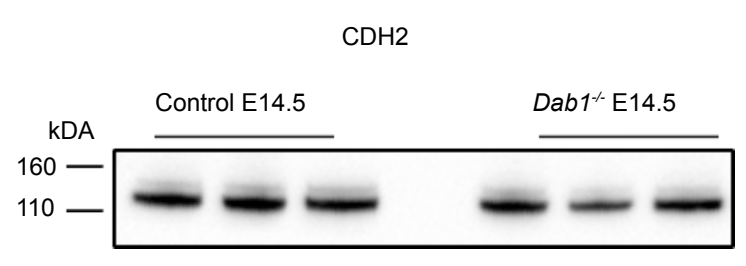

B

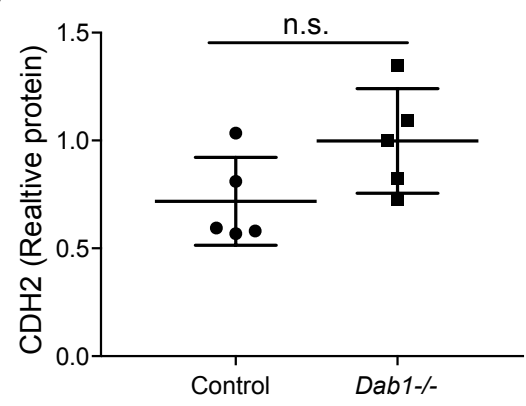

Figure 10 - Figure supplement 1. CDH2 expression levels in the ventral midbrain of control and Dab1\% brains at E14.5. Immuno blots for ventral midbrain tissue from control and Dab1/- brains $(A)$ show no changes in relative protein levels of $\mathrm{CDH} 2$ in Dab11- tissue compared to controls (B). $p$-value 0.08 as assessed by Student's t-test ( $n=5$ brains/genotype).

Movie 1. Time lapse imaging with 2 photon excitation of ex vivo embryonic slices of the ventral midbrain.

Time-lapse imaging of control (left) and Dab1\% (right) organotypic slices with mosaic labelling of $\mathrm{SN}-\mathrm{mDA}$ neurons reveal aberrant orientation and slower migration of $\mathrm{Dab} 1^{1-}$ mDA neurons.

Movie 2. SN-mDA neurons display dynamic cell morphology.

$3 \mathrm{D}$ projection of a transitionary $\mathrm{mDA}$ neurons at $\mathrm{t}=0 \mathrm{~min}\left(360^{\circ}\right.$ rotation) followed by MIP frames of the same neuron at subsequent time-points. Migratory spurts only occur in bipolar morphology while cell remains stationary or displays slow migration during multipolar phase.

Movie 3. Morphology as detected by YFP mosaic labelling is similar to morphology detected by TH antibody.

Example SN-mDA neuron from fixed, cleared whole-mount embryonic brain of the same age as used in time-lapse experiments (E14.5) shows similar morphology with YFP (green) and TH (magenta) immunostaining.

\section{References}

Alcántara S, Ruiz M, D’Arcangelo G, Ezan F, Lecea L, Curran T, et al. Regional and Cellular Patterns of reelin mRNA Expression in the Forebrain of the Developing and Adult Mouse. Journal of Neuroscience. 1998; 18(19):7779-7799. http://doi.org/10.1523/JNEUROSCI.18-19-07779.1998. 
Arioka Y, Shishido E, Kubo H, Kushima I, Yoshimi A, Kimura H. Single-cell trajectory analysis of human homogenous neurons carrying a rare RELN variant. Translational Psychiatry. 2018; p. 1-12.

Berens P. CircStat: A MATLAB Toolbox for Circular Statistics. Journal of Statistical Software. 2009; 31(10). http://doi.org/10.18637/jss.v031.i10.

Bifsha P, Balsalobre A, Drouin J. Specificity of Pitx3-Dependent Gene Regulatory Networks in Subsets of Midbrain Dopamine Neurons. . 2017; 54:4921-4935. https://doi.org/10.1007/s12035-016-0040-y.

Björklund A, Dunnett SB. Dopamine neuron systems in the brain: an update. Trends in Neurosciences. 2007; 30(5):194-202. http://doi.org/10.1016/j.tins.2007.03.006.

Blaess S, Ang SL. Genetic control of midbrain dopaminergic neuron development. Wiley Interdisciplinary Reviews: Developmental Biology. 2015; 4(2):113-134. http://doi.org/10.1002/wdev.169.

Blaess S, Bodea GO, Kabanova A, Chanet S, Mugniery E, Derouiche A. Temporal-spatial changes in Sonic Hedgehog expression and signaling reveal different potentials of ventral mesencephalic progenitors to populate distinct ventral midbrain nuclei. Neural Development. 2011; 6(1):29. http://doi.org/10.1186/1749-81046-29.

Bodea GO, Blaess S. Organotypic Slice Cultures of Embryonic Ventral Midbrain: A System to Study Dopaminergic Neuronal Development <em>in vitro</em>. Journal of Visualized Experiments. 2012; 59:1-7. http://doi.org/10. $3791 / 3350$.

Bodea GO, Spille JH, Abe P, Andersson AS, Acker-Palmer A, Stumm R, et al. Reelin and CXCL12 regulate distinct migratory behaviors during the development of the dopaminergic system. Development. 2014; 141(3):661673. http://doi.org/10.1242/dev.099937.

Britto JM, Tait KJ, Johnston LA, Hammond VE, Kalloniatis M, Tan SS. Altered speeds and trajectories of neurons migrating in the ventricular and subventricular zones of the reeler neocortex. Cerebral Cortex. 2011; 21(5):1018-1027. http://doi.org/10.1093/cercor/bhq168.

Britto JM, Tait KJ, Lee EP, Gamble RS, Hattori M, Tan SS. Exogenous Reelin Modifies the Migratory Behavior of Neurons Depending on Cortical Location. Cerebral Cortex. 2013; 24(11):2835-2847. http://doi.org/10.1093/cercor/ bht123.

Chai X, Förster E, Zhao S, Bock HH, Frotscher M. Reelin stabilizes the actin cytoskeleton of neuronal processes by inducing $n$-cofilin phosphorylation at serine3. Journal of Neuroscience. 2009; 29(1):288-299. https: //doi.org/10.1523/JNEUROSCI.2934-08.2009.

Chai X, Zhao S, Fan L, Zhang W, Lu X, Shao H, et al. Reelin and Cofilin Cooperate During the Migration of Cortical Neurons: a Quantitative Morphological Analysis. Development. 2016; 143:1029-1040. doi:10.1242/dev.134163.

Ekstrand MI, Terzioglu M, Galter D, Zhu S, Hofstetter C, Lindqvist E, et al. Progressive parkinsonism in mice with respiratory-chain-deficient dopamine neurons. Proceedings of the National Academy of Sciences of the U S A. 2007; 104(4):1325-1330. 10.1073/pnas.0605208103; http://doi.org/10.1073/pnas.0605208103.

Fishell G, Kooy D. Pattern formation in the striatum: developmental changes in the distribution of striatonigral neurons. Journal of Neuroscience. 1987; 7(1969-1978):10-1523. DOI:https://doi.org/10.1523/JNEUROSCI.07-0701969.1987.

Franco SJ, Martinez-Garay I, Gil-Sanz C, Harkins-Perry SR, Müller U. Reelin Regulates Cadherin Function via Dab1/Rap1 to Control Neuronal Migration and Lamination in the Neocortex. Neuron. 2011; 69(3):482-497. https://doi.org/10.1016/j.neuron.2011.01.003; http://doi.org/10.1016/j.neuron.2011.01.003, via Dab1/Rap1 to.

Frotscher M, Zhao S, Wang S, Chai X. Reelin Signaling Inactivates Cofilin to Stabilize the Cytoskeleton of Migrating Cortical Neurons. Frontiers in Cellular Neuroscience. 2017; 11:805-7. https://doi.org/10.3389/fncel.2017.00148.

Gärtner A, Fornasiero EF, Munck S, Vennekens K, Seuntjens E, Huttner WB. N-cadherin specifies first asymmetry in developing neurons. The EMBO Journal. 2012; 31(8):1893-1903. http://doi.org/10.1038/emboj.2012.41.

Gil-Sanz C, Franco SJ, Martinez-Garay I, Espinosa A, Harkins-Perry S, Müller U. Cajal-Retzius Cells Instruct Neuronal Migration by Coincidence Signaling between Secreted and Contact-Dependent Guidance Cues. Neuron. 2013; 79(3):461-477. http://doi.org/10.1016/j.neuron.2013.06.040.

Grace AA, Bunney BS. Nigral dopamine neurons: intracellular recording and identification with L-dopa injection and histofluorescence. Science. 1980; 210(4470):654-656. http://doi.org/10.1126/science.7433992. 
Hack I, Bancila M, Loulier K, Carroll P, Cremer H. Reelin is a detachment signal in tangential chain-migration during postnatal neurogenesis. Nature Neuroscience. 2002; 5(10):939-945. http://doi.org/10.1038/nn923.

Harfe BD, Scherz PJ, Nissim S, Tian H, McMahon AP, Tabin CJ. Evidence for an Expansion-Based Temporal Shh Gradient in Specifying Vertebrate Digit Identities. Cell. 2004; 118(4):517-528. http://doi.org/10.1016/j.cell.2004.07. 024.

Hartfuss E, Förster E, Bock HH, Hack MA, Leprince P, Luque JM. Reelin signaling directly affects radial glia morphology and biochemical maturation. Development. 2003; 130(19):4597-4609. http://doi.org/10.1242/dev. 00654.

Hiesberger T, Trommsdorff M, Howell BW, Goffinet A, Mumby MC, Cooper JA, et al. Direct binding of Reelin to VLDL receptor and ApoE receptor 2 induces tyrosine phosphorylation of disabled- 1 and modulates tau phosphorylation. Neuron. 1999; 24(2):481-489. https://doi.org/10.1016/S0896-6273(00)80861-2.

Howell BW, Hawkes R, Soriano P, Cooper JA. Neuronal position in the developing brain is regulated by mouse disabled-1. Nature. 1997; 389(6652):733-737. http://doi.org/10.1038/39607.

Izumi Y, Wakita S, Kanbara C, Nakai T, Akaike A, Kume T. Integrin $\alpha 5 \beta 1$ expression on dopaminergic neurons is involved in dopaminergic neurite outgrowth on striatal neurons. Scientific Reports. 2017; 7. http://doi.org/10. 1038/srep42111.

Jaqaman K, Loerke D, Mettlen M, Kuwata H, Grinstein S, Schmid SL, et al. Robust single-particle tracking in live-cell time-lapse sequences. Nature Methods. 2008; 5(8):695-702. http://doi.org/10.1038/nmeth.1237.

Jossin Y, Cooper JA. Reelin, Rap1 and N-cadherin orient the migration of multipolar neurons in the developing neocortex. Nature Neuroscience. 2011; 14(6):697-703. http://doi.org/10.1038/nn.2816.

Jossin Y, Goffinet AM. Reelin Signals through Phosphatidylinositol 3-Kinase and Akt To Control Cortical Development and through mTor To Regulate Dendritic Growth. Molecular and Cellular Biology. 2007; 27(20):7113-7124. http://doi.org/10.1128/MCB.00928-07.

Kang WY, Kim SS, Cho SK, Kim S, Suh-Kim H, Lee YD. Migratory Defect of Mesencephalic Dopaminergic Neurons in Developing Reeler mice. Anatomy \& Cell Biology. 2010; 43(3):241-11. https://doi.org/10.5115/acb.2010.43.3.241.

Keilani S, Sugaya K. Reelin induces a radial glial phenotype in human neural progenitor cells by activation of Notch-1. BMC Developmental Biology. 2008; 8(1):69. http://doi.org/10.1186/1471-213X-8-69.

Kriegstein AR, Noctor SC. Patterns of neuronal migration in the embryonic cortex. Trends in Neurosciences. 2004; 27(7):392-399. http://doi.org/10.1016/j.tins.2004.05.001.

Krüger MT, Zhao S, Chai X, Brunne B, Bouché E, Bock HH, et al. Role for Reelin-induced cofilin phosphorylation in the assembly of sympathetic preganglionic neurons in the murine intermediolateral column. The European Journal of Neuroscience. 2010; 32(10):1611-1617. http://doi.org/10.1111/j.1460-9568.2010.07433.x.

Leemhuis J, Bouché E, Frotscher M, Henle F, Hein L, Herz J. Reelin signals through apolipoprotein E receptor 2 and Cdc42 to increase growth cone motility and filopodia formation. . Journal of Neuroscience. 2010; 30(44):14759-14772. http://doi.org/10.1523/JNEUROSCI.4036-10.2010.

Li J, Duarte T, Kocabas A, Works M, McConnell SK, Hynes MA. Evidence for topographic guidance of dopaminergic axons by differential Netrin-1 expression in the striatum. Molecular and Cellular Neuroscience. 2014; 61:85-96. https://doi.org/10.1016/j.men.2014.05.003.

Longair MH, Baker DA, Armstrong JD. Simple Neurite Tracer: Open Source software for reconstruction, visualization and analysis of neuronal processes. Bioinformatics. Bioinformatics. 2011; .

Maciver SK, Hussey PJ. The ADF/cofilin family: actin-remodeling proteins. Genome Biology. 2002; 3(5):3007-1. http://doi.org/10.1186/gb-2002-3-5-reviews3007.

Manno GL, Gyllborg D, Codeluppi S, Nishimura K, Saltó C, Zeisel A. Molecular Diversity of Midbrain Development in Mouse, Human, and Stem Cells. Cell. 2016; 167(2):566-580. http://doi.org/10.1016/j.cell.2016.09.027.

Martini FJ, Valiente M, Bendito GL, Szabo G, Moya F, Valdeolmillos M, et al. Biased Selection of Leading Process Branches Mediates Chemotaxis During Tangential Neuronal Migration. Development. 2008; 136(1):41-50.

Morales M, Margolis EB. Ventral tegmental area: cellular heterogeneity, connectivity and behaviour. Nature Reviews Neuroscience. 2017; 18(2):73-85. http://doi.org/10.1038/nrn.2016.165. 
Nishikawa S, Goto S, Yamada K, Hamasaki T, Ushio Y. Lack of Reelin causes malpositioning of nigral dopaminergic neurons: Evidence from comparison of normal andRelnrl mutant mice. The Journal of Comparative Neurology. 2003; 461(2):166-173. http://doi.org/10.1002/cne.10610.

Panman L, Papathanou M, Laguna A, Oosterveen T, Volakakis N, Acampora D. Sox6 and Otx2 Control the Specification of Substantia Nigra and Ventral Tegmental Area Dopamine Neurons. CellReports. 2014; 8(4):10181025. http://doi.org/10.1016/j.celrep.2014.07.016.

Park TJ, Curran T. Crk and Crk-Like Play Essential Overlapping Roles Downstream of Disabled-1 in the Reelin Pathway. Journal of Neuroscience. 2008; 28(50):13551-13562. http://doi.org/10.1523/JNEUROSCI.4323-08.2008.

Petrie RJ, Doyle AD, Yamada KM. Random versus directionally persistent cell migration. Nature Reviews Molecular Cell Biology. 2009; 10(8):538-549. http://doi.org/10.1038/nrm2729.

Phelps P. Evidence for a Cell-Specific Action of Reelin in the Spinal Cord. Developmental Biology. 2002; 244(1):180-98.

Poulin JF, Zou J, Drouin-Ouellet J, Kim KYA, Cicchetti F, Awatramani RB. Defining Midbrain Dopaminergic Neuron Diversity by Single-Cell Gene Expression Profiling. Cell Reports. 2014; 9(3):930-943. http://doi.org/10.1016/j.celrep. 2014.10.008.

Prakash N, Puelles E, Freude K, Trümbach D, Omodei D, Salvio MD. Nkx6-1 controls the identity and fate of red nucleus and oculomotor neurons in the mouse midbrain. Development. 2009; 136(15):2545-2555. http://doi.org/10.1242/dev.031781.

Przedborski S. The two-century journey of Parkinson disease research. Nature Reviews Neuroscience. 2017; 18(4):251-259. http://doi.org/10.1038/nrn.2017.25.

Salvio MD, Giovannantonio LGD, Omodei D, Acampora D, Simeone A. Otx2 expression is restricted to dopaminergic neurons of the ventral tegmental area in the adult brain. The International Journal of Developmental Biology. 2010; 54(5):939-945. http://doi.org/10.1387/ijdb.092974ms.

Schwarz MK, Scherbarth A, Sprengel R, Engelhardt J, Theer P, Giese G. Fluorescent-Protein Stabilization and High-Resolution Imaging of Cleared, Intact Mouse Brains. PLoS ONE. 2015; 10(5):0124650-26. http: //doi.org/10.1371/journal.pone.0124650.

Sekine K, Kawauchi T, Kubo Kl, Honda T, Herz J, Hattori M. Reelin Controls Neuronal Positioning by Promoting Cell-Matrix. Neuron. 2012; 76(2):353-369. http://doi.org/10.1016/j.neuron.2012.07.020.

Sekine K, Kubo KI, Nakajima K. How does Reelin control neuronal migration and layer formation in the developing mammalian neocortex? Neuroscience Research. 2014; 86:50-58. http://doi.org/10.1016/j.neures.2014. 06.004.

Sharaf A, Bock HH, Spittau B, Bouché E, Krieglstein K. ApoER2 and VLDLr Are Required for Mediating Reelin Signalling Pathway for Normal Migration and Positioning of Mesencephalic Dopaminergic Neurons. PLoS ONE. 2013; 8(8):71091-11. http://doi.org/10.1371/journal.pone.0071091.

Sharaf A, Rahhal B, Spittau B, Roussa E. Localization of Reelin Signaling Pathway Components in Murine Midbrain and Striatum. Cell and Tissue Research. 2015; 359(2):393-407. https://doi.org/10.1007/s00441-014-2022-6.

Simó S, Jossin Y, Cooper JA. Cullin 5 regulates cortical layering by modulating the speed and duration of Dab1-dependent neuronal migration. Journal of Neuroscience. 2010; 30(16):5668-5676. http://doi.org/10.1523/ JNEUROSCI.0035-10.2010.

Srinivas S, Watanabe T, Lin CS, William CM, Tanabe Y, Jessell TM, et al. Cre reporter strains produced by targeted insertion of EYFP and ECFP into the ROSA26 locus. BMC Developmental Biology. 2001; 1(1):4. http://doi.org/10.1186/1471-213X-1-4.

Tinevez JY, Perry N, Schindelin J, Hoopes GM, Reynolds GD, Laplantine E. TrackMate: An open and extensible platform for single-particle tracking. Methods. 2017; 115:80-90. http://doi.org/10.1016/j.ymeth.2016.09.016.

Trommsdorff M, Gotthardt M, Hiesberger T, Shelton J, Stockinger W, Nimpf J, et al. Reeler/Disabled-like disruption of neuronal migration in knockout mice lacking the VLDL receptor and ApoE receptor 2. Cell. 1999; 97(6):689-701. https://doi.org/10.1016/S0092-8674(00)80782-5.

Vaswani AR, Blaess S. Reelin Signaling in the Migration of Ventral Brain Stem and Spinal Cord Neurons. Frontiers Cellular Neuroscience. 2016; 10:579-14. http://doi.org/10.3389/fncel.2016.00062. 
Volkow ND, Morales M. The Brain on Drugs: From Reward to Addiction. Cell. 2015; 162(4):712-725. http: //doi.org/10.1016/j.cell.2015.07.046.

Voss AK, Britto JM, Dixon MP, Sheikh BN, Collin C, Tan SS, et al. C3G regulates cortical neuron migration, preplate splitting and radial glial cell attachment. Development. 2008; 135(12):2139-2149. http://doi.org/10.1242/dev. 016725 .

Wang S, Brunne B, Zhao S, Chai X, Li J, Lau J. Trajectory Analysis Unveils Reelin's Role in the Directed Migration of Granule Cells in the Dentate Gyrus. . Journal of Neuroscience. 2018; 38(1):137-148. https://doi.org/10.1523/ JNEUROSCI.0988-17.2017.

Weisenhorn DMV, Giesert F, Wurst W. Diversity Matters-Heterogeneity of Dopaminergic Neurons in the Ventral Mesencephalon and Its Relation to Parkinson's Disease. Journal of Neurochemistry. 2016; 139(S1):826. http://doi:10.1111/jnc.13670.

Wu PH, Giri A, Wirtz D. Statistical analysis of cell migration in 3D using the anisotropic persistent random walk model. Nature Protocols. 2015; 10(3):517-527. http://doi.org/10.1038/nprot.2015.030.

Xu B, Goldman JS, Rymar VV, Forget C, Lo PS, Bull SJ. Critical Roles for the Netrin Receptor Deleted in Colorectal Cancer in Dopaminergic Neuronal Precursor Migration, Axon Guidance, and Axon Arborization. Neuroscience. 2010; 169(2):932-949. http://doi.org/10.1016/j.neuroscience.2010.05.025.

Yabut O, Renfro A, Niu S, Swann JW, Marín O, D'Arcangelo G. Abnormal laminar position and dendrite development of interneurons in the reeler forebrain. Brain Research. 2007; 1140:75-83. http://doi.org/10.1016/j.brainres. 2005.09.070.

Zhang JH, Zhao YF, He XX, Zhao Y, He ZX, Zhang L. Dab1 Phosphorylation Participates in the Multipolar-to-Bipolar Transition of Migrating Neurons. CellReports. 2018; 22(13):3598-3611. http://doi.org/10.1016/j.celrep.2018.03.005. 